\title{
Optimization of low aspect ratio, iron dominated dipole magnets
}

\author{
N. Majernik, ${ }^{1,2, *}$ S. K. Barber, ${ }^{2}$ J. van Tilborg, ${ }^{2}$ J. B. Rosenzweig, ${ }^{1}$ and W. P. Leemans ${ }^{2,3}$ \\ ${ }^{1}$ University of California-Los Angeles, Los Angeles, California 90095, USA \\ ${ }^{2}$ Lawrence Berkeley National Lab, Berkeley, California 94720, USA \\ ${ }^{3}$ Deutsches Elektronen Synchrotron DESY, 22607 Hamburg, Germany
}

(Received 23 December 2018; published 27 March 2019)

\begin{abstract}
A study of the optimization of iron dominated dipole magnets with pole face widths comparable or less than the gap size, i.e., low aspect ratio (AR), is conducted using both theoretical and computational approaches. This regime of magnet design is particularly relevant in the context of laser plasma accelerators (LPA) due to unique beam parameters and geometric constraints, namely large energy spreads and the requirement for large apertures to accommodate drive laser passage. The breakdown of commonly employed approximations and rules of thumb in typical AR $\gg 1$ magnet design is examined. A library of generalized, optimized pole face geometries is provided to expedite optimization of future magnets. Finally, this methodology is used to design an electromagnetic chicane which has been fabricated, validated, and is currently in use in an x-ray free electron laser driven LPA experiment at LBNL.
\end{abstract}

DOI: 10.1103/PhysRevAccelBeams.22.032401

\section{INTRODUCTION}

Many disciplines employ dipole magnets, often requiring a consistent, flat field over some volume of interest. These include beam [1-5], plasma [6], and AMO (atomic, molecular, and optical) physics [7] as well as myriad applications in chemistry [8,9], engineering [10], and medicine [11,12]. A particular subclass of interest is that of iron-dominated magnets, in contrast with coil dominated, e.g., superconducting and air cored, wherein the field in the region of interest is shaped by a ferromagnetic yoke and, most importantly, pole face. In these magnets, the high permeability of ferromagnetic materials (typically iron-based) is used to confine, direct, and amplify the magnetic flux from a source, generally coils of wire or permanent magnets. To produce a larger volume of flat field, the most obvious approach is to simply increase the size of the yoke and pole faces. In some applications, this approach (with minor refinements discussed below) is sufficient. This work will discuss the optimization of field quality for a fixed magnet size where the gap between the pole faces is of the same order as the width of the pole face- the case of low aspect ratio. Such magnets are especially useful in the rapidly advancing field of laser plasma accelerators [2,13-15] where the width of

\footnotetext{
*Corresponding author. NMajernik@ucla.edu

Published by the American Physical Society under the terms of the Creative Commons Attribution 4.0 International license. Further distribution of this work must maintain attribution to the author(s) and the published article's title, journal citation, and DOI.
}

good field region required might be low but the gap between pole faces needs to be large to accommodate the large beam pipes required for downstream transport of the drive laser.

\section{THEORETICAL TREATMENT}

\section{A. Multipole decomposition}

A brief derivation of the multipole convention follows, with a more thorough explanation found in Ref. [1]. Maxwell's equations for magnetostatics in the absence of sources are

$$
\begin{aligned}
\boldsymbol{\nabla} \times \mathbf{B} & =0, \\
\boldsymbol{\nabla} \cdot \mathbf{B} & =0 .
\end{aligned}
$$

One may define a vector field $\mathbf{F}=\mathbf{A}+i V$ such that $\mathbf{B}=\boldsymbol{\nabla} \times \mathbf{A}=-\boldsymbol{\nabla} V$. In the $2 \mathrm{D}$, current free case, it can be shown that if $\mathbf{F}$ is written as a function of $z=x+i y$, then $\mathbf{F}$ satisfies the Maxwell conditions by construction. The real part of $\mathbf{F}$ gives the flux lines while the imaginary part gives the equipotentials. One particularly useful definition which expresses $\mathbf{F}$ as $\sum C_{n} z^{n}$ is called the multipole decomposition. In the multipole decomposition $n=1$ refers to the dipole component, $n=2$ is the quadrupole component, $n=3$ is the sextupole component, and so on. A commonly employed approximation is to treat the iron elements of a system as having infinite permeability. This means that the pole face surface is an equipotential surface and that conformal mapping can transform an infinite pole face and its concomitant perfect, infinite dipole field into the fields of a finite pole [16]. This approximation is generally insufficient for real world 
magnet design due to the finite permeability of iron, coil fields, saturation, and other considerations. Therefore, it tends to only be a useful starting point for refinement in finite element method (FEM) simulations.

\section{B. Flat and shimmed poles}

The most common way to improve the good field region for a given aspect ratio (AR) is by shimming the pole face by placing small, numerically optimized bumps at the edges of the face. Expressions of the form

$$
\frac{a}{h}=c_{1} \ln \left(\frac{\Delta B}{B}\right)+c_{2},
$$

are given in [1], where $a$ is the overhang of the pole face extending beyond the good field region, $h$ is the gap between the magnet midplane and the pole face, and $\Delta B / B$ is the maximum allowable, normalized field error. Coefficients $c_{1}$ and $c_{2}$ for both shimmed and flat cases are provided, giving a relationship between the magnet geometry and the field quality required. We define the term good field region, $x_{\mathrm{gfr}}$, as the width on axis of the good field region satisfying good field quality, GFQ $=B / \Delta B$. By writing

$$
\mathrm{AR}=\frac{a+x_{\mathrm{gfr}}}{h},
$$

Eq. (3) can be rearranged as

$$
\begin{aligned}
\mathrm{AR}_{\text {unopt }} & =\frac{x_{\mathrm{gfr}}}{h}-0.9-0.36 \ln \left(\frac{1}{\mathrm{GFQ}}\right) \\
\mathrm{AR}_{\mathrm{opt}} & =\frac{x_{\mathrm{gfr}}}{h}-0.25-0.14 \ln \left(\frac{1}{\mathrm{GFQ}}\right),
\end{aligned}
$$

with the provided coefficients for the unoptimized flat case and optimized shim case respectively.

Although it was not made explicit by the analysis of Tanabe, these equations break down for magnets where the gap is comparable or large compared to the pole width (detailed below). This divergence motivated the search for improved pole face designs for low AR magnets.

\section{Parabolic poles}

The symmetry conditions of a particular magnet design will impose boundary conditions which forbid certain multipole components; the remaining, allowed components comprise the error spectrum. The symmetry constraint on the $n$th moment for a $N$ th order magnet is

$$
F_{n}\left(\theta+\frac{\pi}{N}\right)=-F_{n}(\theta) .
$$

There is an extended discussion of the application of Eq. (5) in Ref. [1] but we will only concern ourselves

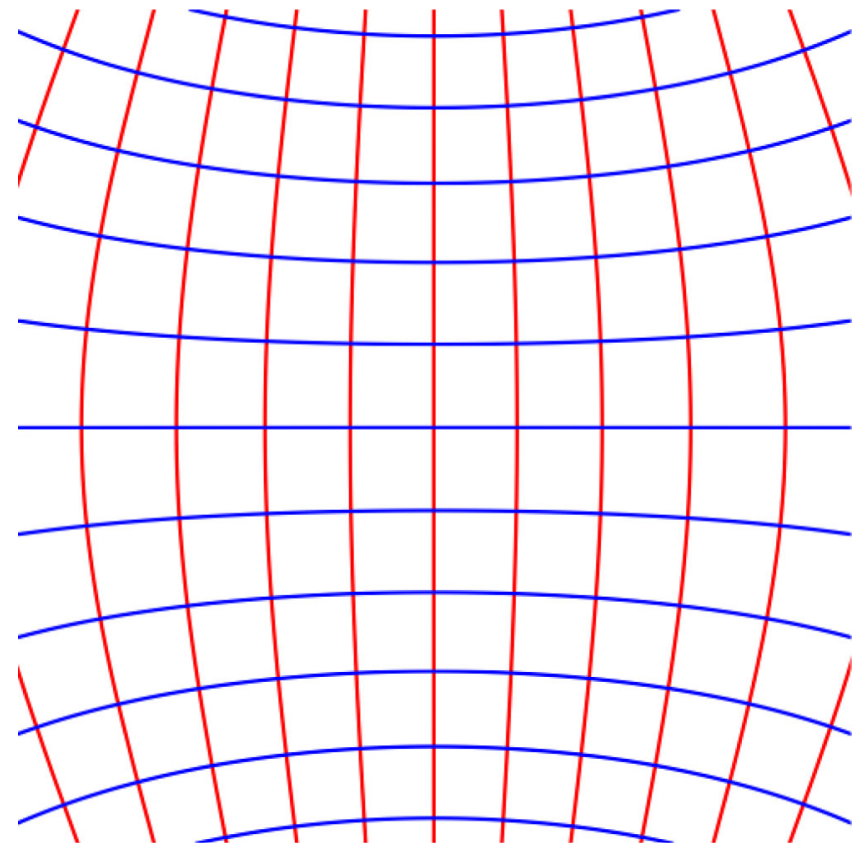

FIG. 1. Field lines (red) and equipotentials (blue) for a dipole magnet with small virtual sextupole component.

with the dipole case which has allowed multipole moments $n=3,5,7,9 \ldots$ Therefore, the lowest order error which needs to be counteracted is $n=3$, or sextupole. In a dipole with finite, flat pole faces, this manifests as a "virtual sextupole" which causes the field lines to bow out, away from the center. This is illustrated in Fig. 1 for $C_{1} z+C_{3} z^{3}$.

Note that the equipotentials bow toward the center. This can be counteracted by curving the pole face in a manner equal and opposite to this equipotential curve, strengthening the field near the edges to reduce the virtual sextupole. By selecting a parabolic profile with an even greater curvature, intrinsic sextupole focusing can be realized in a linear undulator [17]. The optimization described below uses this approach to seed the final FEM optimization which will account for considerations left out of this approximation.

For example, consider an $\mathrm{AR}=1$ dipole, shown in Fig. 2. After a multipole decomposition, normalizing to the dipole coefficient (i.e., $C_{1}=1$ ), the sextupole contribution is $C_{3}=0.035$ inside a 0.5 gap unit radius circle on centerline. The corresponding field lines and equipotentials from only these lowest order components are shown in Fig. 3.

The equipotential line which is nearest to the pole face is found and is approximated with a parabola, in this case with equation $y=-0.10 x^{2}-0.96$. Construct new pole faces with parabolic component equal and opposite to this isocontour curvature (Fig. 4).

Calculating the multipole components as before, find that $C_{3}$ has been reduced by more than an order of magnitude 


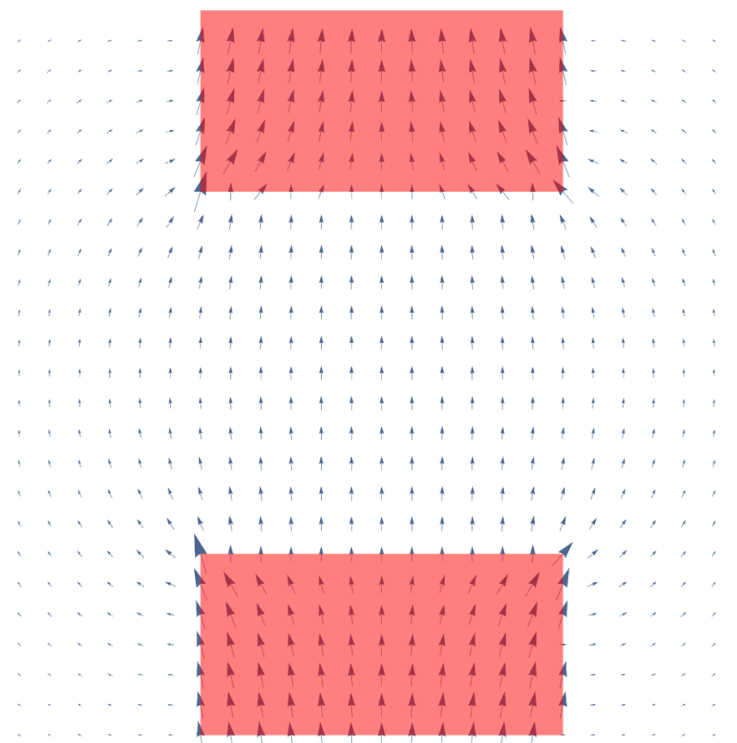

FIG. 2. $A R=1$ dipole with flat pole faces (red) with magnetic field vector plot.

to 0.003 . This serves as a very good starting point for final FEM optimization to tune out higher order effects.

\section{Splined poles}

Using splines to define the pole face permits the creation of poles with arbitrary levels of complexity. Splines have been employed in the optimization of axisymmetric MRI

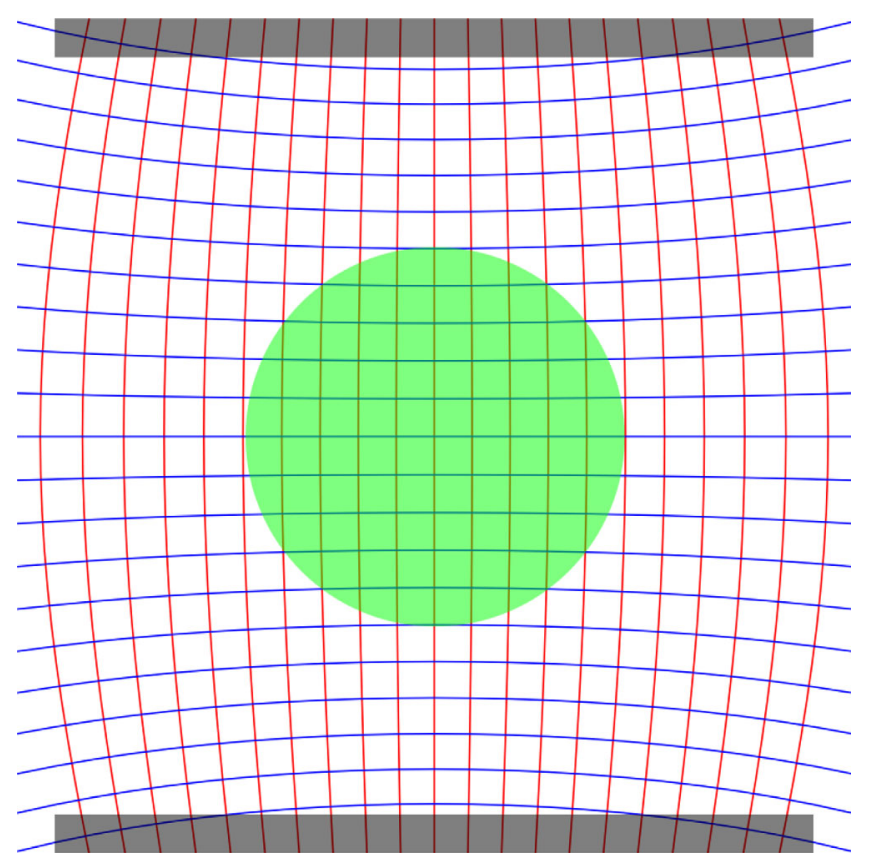

FIG. 3. Field lines (red) and equipotentials (blue) for a $C_{1}=1$, $C_{3}=0.035$ field. Flat pole faces are shown in grey and the area where the decomposition is calculated is in green.

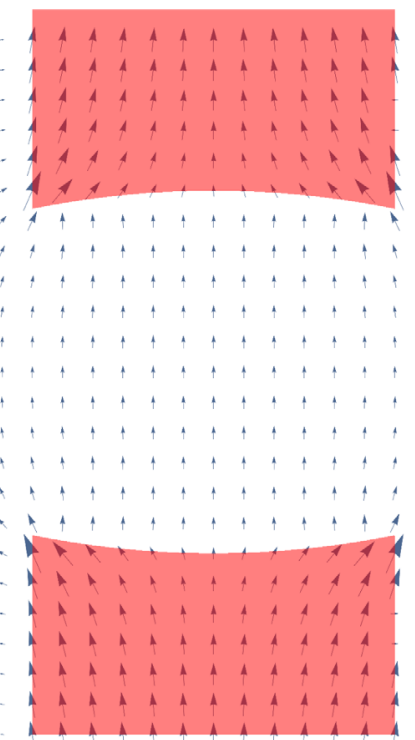

FIG. 4. $\mathrm{AR}=1$ dipole with parabolic $\left( \pm 0.1 x^{2} \mp 0.96\right)$ pole faces (red) with magnetic field vector plot.

magnets $[18,19]$. The details of this current implementation are discussed in the following section.

\section{SIMULATION METHODS}

The parameter space of magnet design is continuous and multidimensional, so for this work a representative, discrete subset is examined which covers magnets which are both physically relevant and germane to the topic at hand. Specifically, we will consider three aspect ratios (AR): $0.5,1$, and 1.5; three good field requirements (GFQ): 1 in $10^{3}, 1$ in $10^{3.5}$, and 1 in $10^{4}$; and five pole face geometries: flat, shimmed, parabolic, three point spline, and five point spline. Figure 5 is a visual aid to the terminology used throughout the paper.

We define here the following terms:

Aspect ratio - the ratio of the pole face gap at the centerline to the width of the pole at its widest;

Good field region —on the horizontal axis, the maximum $x_{\text {gfr }}$ value such that

$$
\begin{aligned}
\frac{|\mathbf{B}(x, 0)-\mathbf{B}(0,0)|}{|\mathbf{B}(0,0)|} & <\frac{1}{\mathrm{GFQ}} \\
\forall x & <x_{\mathrm{gfr}} ;
\end{aligned}
$$

Shimmed - a semicircle protrusion with center a distance one radius from the pole edge;

Spline-a cubic spline interpolation on points at $x=0$, \pm 0.5 , and \pm 1 width units for the three point spline and an interpolation of points at $x=0, \pm 0.25, \pm 0.5, \pm 0.75$, and \pm 1 width units for a five point spline. The point at $x=0$ has $y=1$ gap unit and all other points satisfy $y(x)=y(-x)$. 


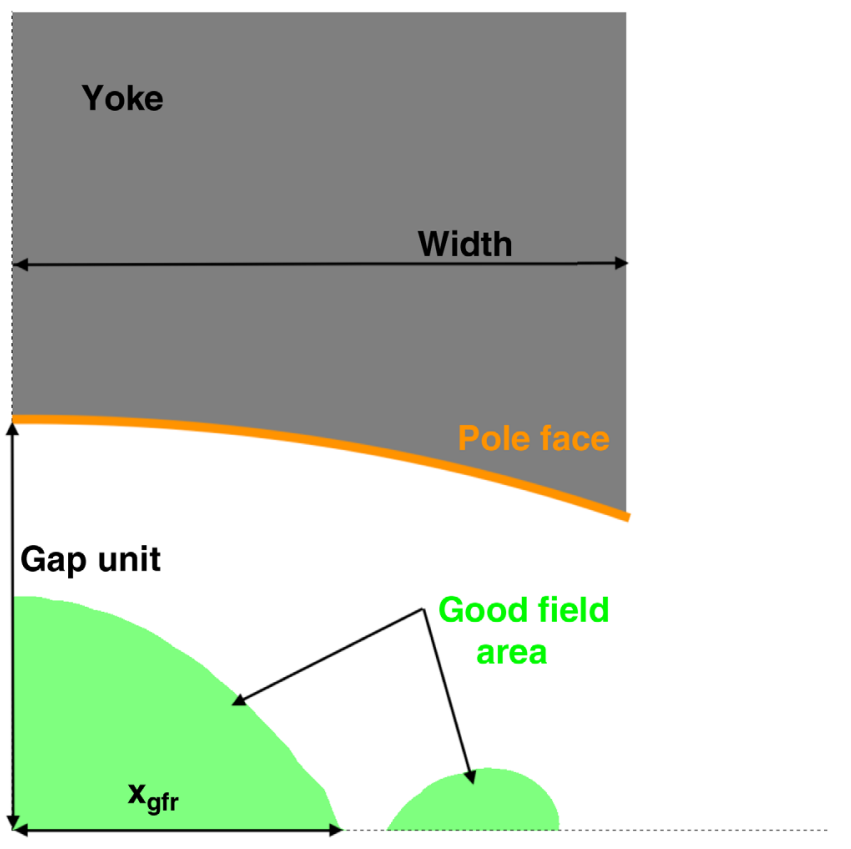

FIG. 5. Visual aid to definitions of geometric features.

All geometries are subject to the additional constraint that they do not intersect a beam pipe with a radius of 0.9 gap units. In all cases (each AR, GFQ, and geometry) all free variables are adjusted to maximize $x_{\mathrm{gfr}}$. In the shimmed and parabolic cases, each with one free variable, this is done with an exhaustive search. In the three- and fivepoint spline cases (with two and four free variables, respectively) this procedure is done using particle swarm optimization (PSO) $[19,20]$. The 2D FEM simulation is done using the program POISSON [21], simulating a single quadrant of an H-magnet, with a mesh step size of 0.01 gap units. The pole face is taken to be far from the rest of the yoke and coils. It is further assumed that the magnet is operating far from saturation and the yoke is made from low carbon steel acting linearly with relative permeability, $\mu / \mu_{0}$, of 4000 . All additional post processing, including the coordination of the particle swarm is done with Mathematica.

\section{SIMULATION RESULTS}

The results for $x_{\text {gfr }}$ given in gap units follow, broken down by GFQ. Tables I-III give the best case results for GFQs $10^{3}$, $10^{3.5}$, and $10^{4}$ respectively. Figures $6-8$ illustrate these results and compare them to theoretical results for shimoptimized and unoptimized pole faces obtained from Eq. (4). In these plots, better performance is indicated by points which are lower (smaller AR for given performance requirement) and further to the right (larger good field region for given GFQ and AR). It should be noted that the comparison to Ref. [1] is imperfect due to the use of relatively low AR; in the more usual, AR $\gg 1$ regime, the "Flat" points should overlap the "Tanabe unoptimized" line and the "Shimmed" points should overlap the "Tanabe optimized" line.
TABLE I. Optimal $x_{\text {gfr }}$ in different geometric configurations with GFQ $=10^{3}$.

\begin{tabular}{lccc}
\hline \hline & $\mathrm{AR}=0.5$ & $\mathrm{AR}=1.0$ & $\mathrm{AR}=1.5$ \\
\hline Flat & 0.05 & 0.09 & 0.21 \\
Round shim & 0.05 & 0.46 & 0.84 \\
Parabolic & 0.07 & 0.47 & 0.69 \\
3 point spline & 0.09 & 0.67 & 1.00 \\
5 point spline & 0.09 & 0.71 & 1.17 \\
\hline \hline
\end{tabular}

TABLE II. Optimal $x_{\text {gfr }}$ in different geometric configurations with GFQ $=10^{3.5}$.

\begin{tabular}{lccc}
\hline \hline & $\mathrm{AR}=0.5$ & $\mathrm{AR}=1.0$ & $\mathrm{AR}=1.5$ \\
\hline Flat & 0.03 & 0.05 & 0.12 \\
Round shim & 0.03 & 0.35 & 0.68 \\
Parabolic & 0.04 & 0.35 & 0.53 \\
3 point spline & 0.05 & 0.57 & 0.83 \\
5 point spline & 0.05 & 0.59 & 1.00 \\
\hline \hline
\end{tabular}

TABLE III. Optimal $x_{\text {gfr }}$ in different geometric configurations with $\mathrm{GFQ}=10^{4}$.

\begin{tabular}{lccc}
\hline \hline & $\mathrm{AR}=0.5$ & $\mathrm{AR}=1.0$ & $\mathrm{AR}=1.5$ \\
\hline Flat & 0.02 & 0.03 & 0.06 \\
Round shim & 0.02 & 0.21 & 0.45 \\
Parabolic & 0.02 & 0.25 & 0.37 \\
3 point spline & 0.03 & 0.41 & 0.57 \\
5 point spline & 0.03 & 0.45 & 0.85 \\
\hline \hline
\end{tabular}

The coefficients used to generate all of these optimizations are in Appendixes A-C. They are provided to give guidance in the optimization of future magnets.

In the cases of flat and shimmed magnets, a clear divergence from the predictions of [1] and Eq. (4) is evident. This breakdown of traditional heuristics in this low aspect ratio regime requires additional care to be taken when designing such magnets. With that said, it should be noted how effective shims are for higher aspect ratio magnets. Between these three GFQ definitions, shimming an $\mathrm{AR}=1$ or $\mathrm{AR}=1.5$ magnet tends to improve $x_{\text {gfr }}$ by a factor of more than four. However, at $\mathrm{AR}=0.5$ there is no improvement from shimming. At $\mathrm{AR}=1$, parabolic faces have nearly identical performance with shims and at $\mathrm{AR}=$ 1.5 shims outperform parabolic faces. In all AR and GFQ cases, three point splines are superior to flat, shimmed, and parabolic faces and five point splines are superior to all other geometries. The superiority of the five point spline is expected since all other geometries are effectively (but not strictly) subsets of this geometry. This comes with the caveat that some of the geometries generated here have sharp corners which might readily saturate in some 


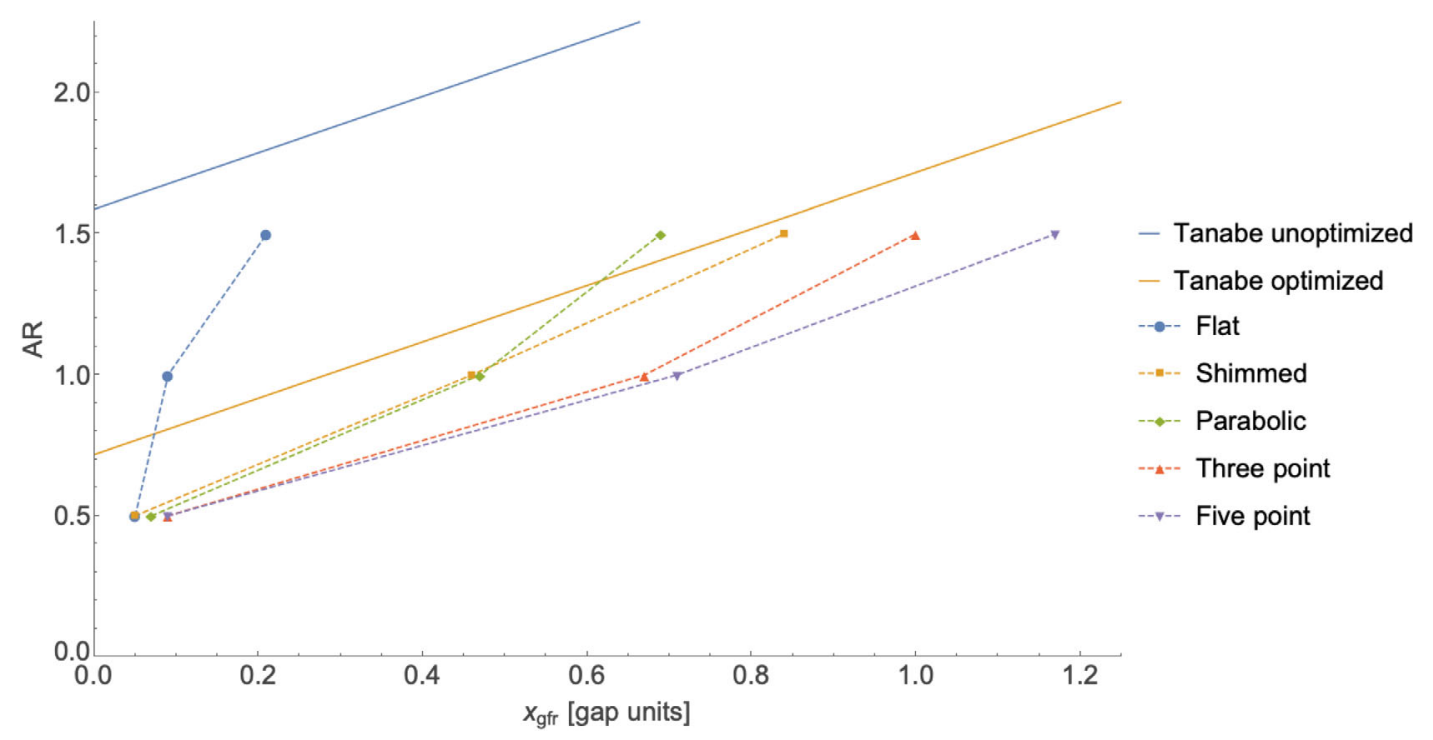

FIG. 6. Optimized simulation results for $\mathrm{GFQ}=10^{3}$ vs [1] estimates. The dashed lines are intended to guide the eye.

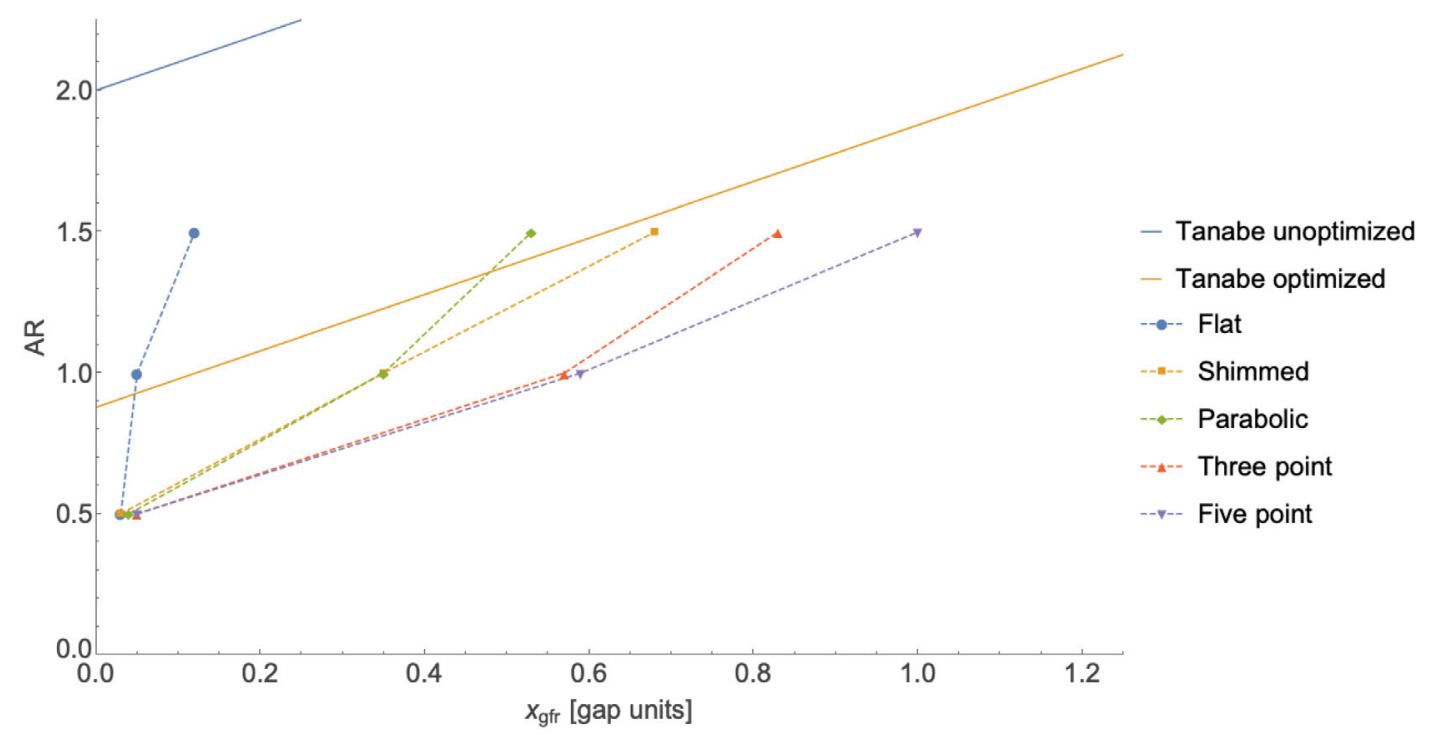

FIG. 7. Optimized simulation results for $\mathrm{GFQ}=10^{3.5}$ vs [1] estimates. The dashed lines are intended to guide the eye.

real-world scenarios. This limitation is discussed in greater detail in the next section.

\section{IMPLEMENTATION}

These theoretical and computational methods were brought to bear on the design of an electromagnetic chicane for an LPA experiment currently being implemented at the LBNL BELLA Laboratory. This experiment aims to demonstrate the generation of coherently enhanced $x$-ray radiation from an LPA driven free electron laser (FEL) [22]. In order to achieve the required slice energy spread, the LPA electron beam must be stretched by a dispersive element, e.g., a chicane. Due to the relatively large energy spread $(\sim 1 \%)$ this can be achieved with minimal sagitta $(\sim 10 \mathrm{~mm})$ but the chicane aperture must accommodate a $50.8 \mathrm{~mm}$ diameter beam pipe since the drive laser must be allowed to propagate downstream for diagnostics. This combination of constraints means that the use of traditional, high aspect ratio dipole designs would require large, heavy, and expensive magnets. By employing the techniques expounded here, an alternative, compact, and low cost design was pursued. For this implementation, the magnet would be run with a sufficiently high field that the "far from saturation" approximation employed above would be violated; this would have led to the saturation of sharp tips suggested by certain spline geometries. This fact, in combination 


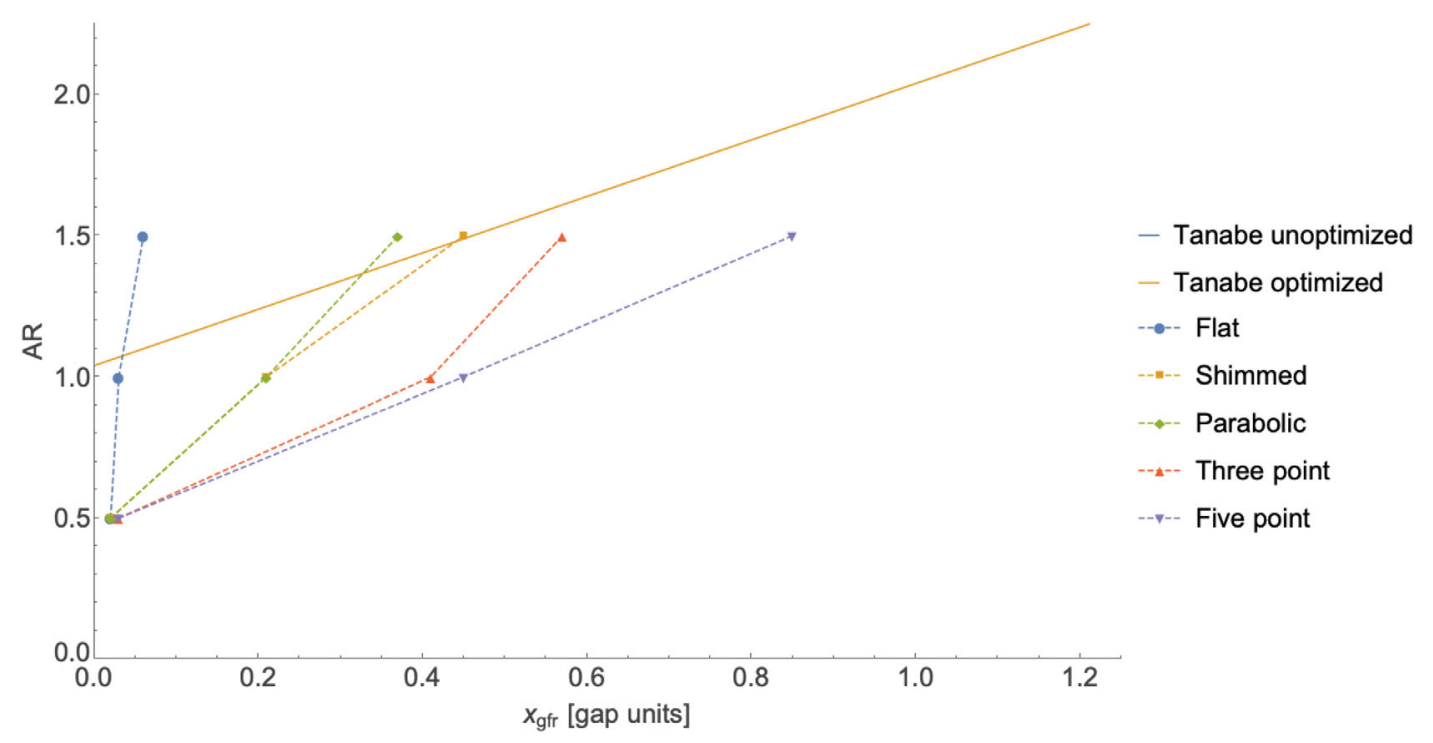

FIG. 8. Optimized simulation results for GFQ $=10^{4}$ vs [1] estimates. The dashed lines are intended to guide the eye.

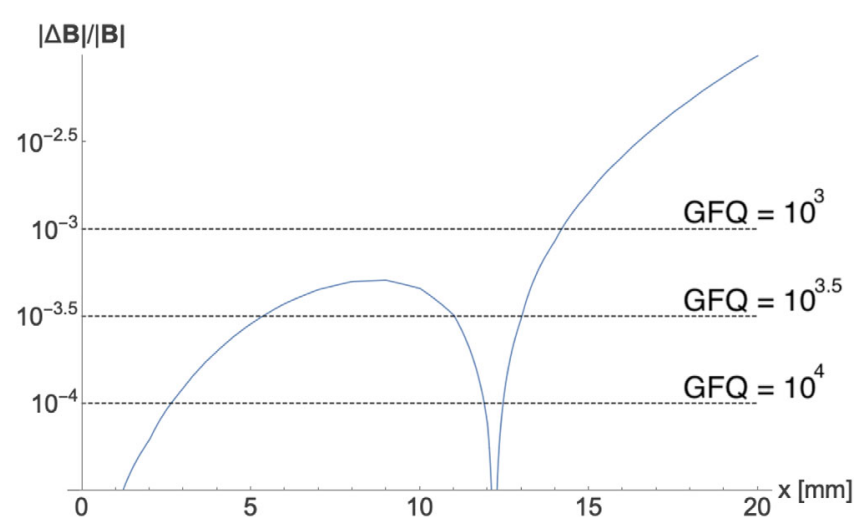

FIG. 9. UCLA-LBNL chicane dipole, relative field error on axis. GFQ levels from previous section shown as dotted lines.

with practical considerations about fabrication, led to the selection of a parabolic pole face.

Each dipole requires a gap unit of $27.3 \mathrm{~mm}$ in order to accommodate a $50.8 \mathrm{~mm}$ diameter beam pipe, translated $5 \mathrm{~mm}$ off axis. We have sought to minimize the pole width subject to this geometric constraint as well as the desire to maintain a GFQ of $>10^{3}$ over an $x_{\text {gfr }}$ of $10 \mathrm{~mm}$ (0.37 gap units) to avoid subjecting the beam to any significant nondipole fields. After optimizing the pole face design to these ends, a pole width of $35 \mathrm{~mm}$ $(\mathrm{AR}=1.37)$ was settled upon; this was projected to give GFQ of $10^{3.3}$ over $x_{\text {gfr }}$ of $13.3 \mathrm{~mm}$ (0.49 gap units), shown in Figs. 9 and 10.

This design was extended to three dimensions and modeled in the magnetostatics code RADIA [23] before generating a mechanical design in Solidworks. This design, along with multiple degree-of-freedom kinematic alignment stage, is shown in Fig. 11. The dipoles were fabricated at

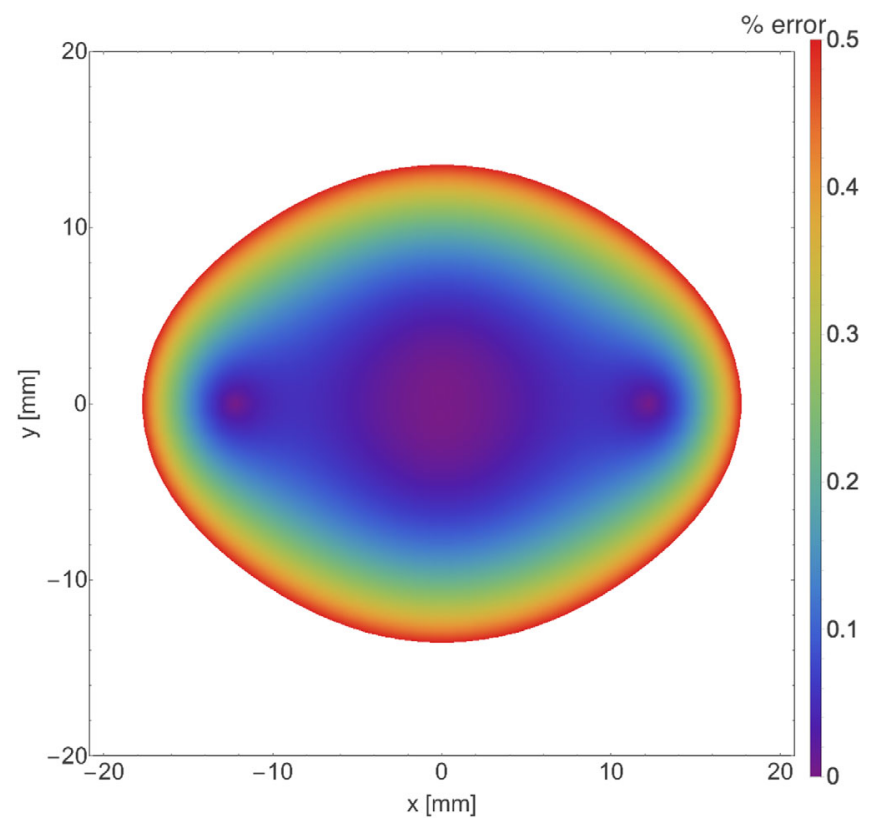

FIG. 10. UCLA-LBNL chicane dipole, relative field error.

UCLA using 1018 steel and nickel plated. The field of each dipole was measured using a purpose built, 3-axis gantry Hall probe and found to conform to the RADIA simulation to within the accuracy of the probe (better than $0.1 \%$ ), giving the requisite field quality specifications. The chicane is presently installed on the experimental beamline to permit beam decompression for the LPA-driven FEL experiment.

\section{CONCLUSION}

An overview of the optimization of dipole magnets with low aspect ratios has been presented. Such magnets are of 


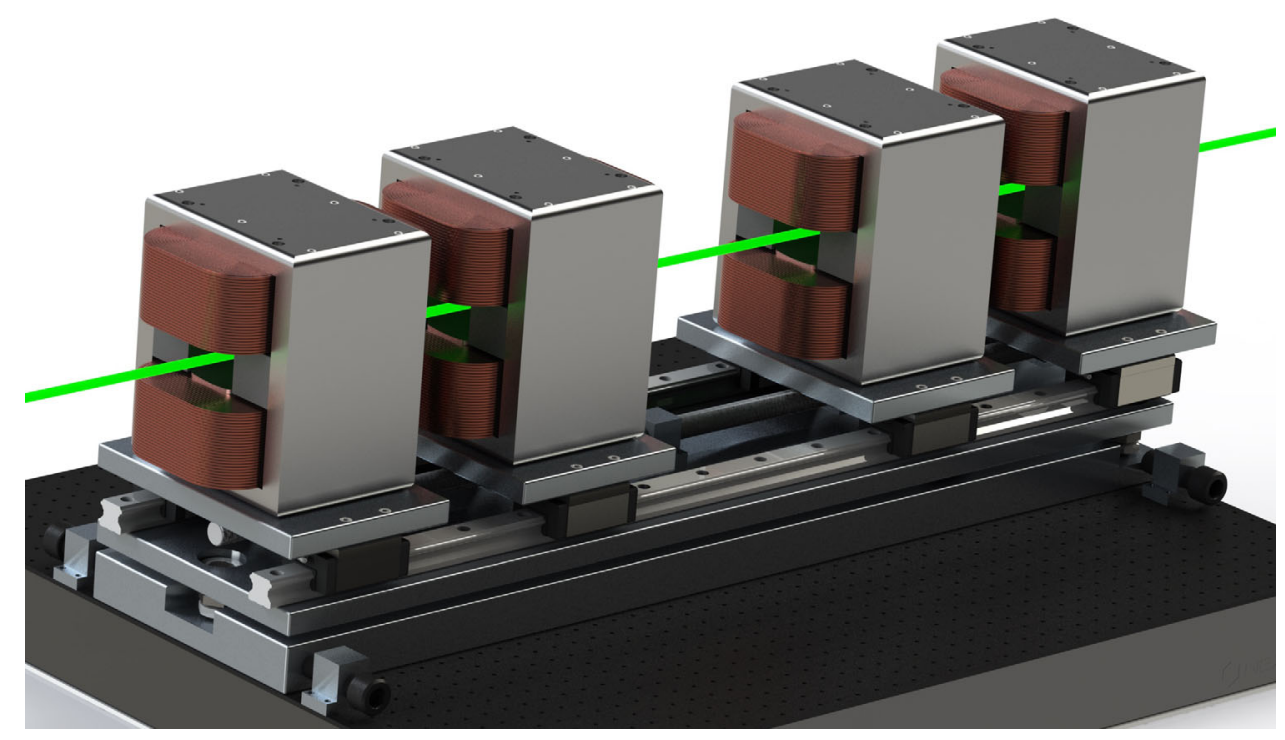

FIG. 11. Rendered model of UCLA-LBNL chicane with kinematic stage.

particular interest to the burgeoning laser plasma accelerator community due to the beam parameters and additional geometric constraints on such experiments. The results suggest that significant improvement in both field quality and area with a given quality can be achieved in this regime by the use of pole faces with more complex features than typical flat or simply shimmed geometries. These theoretical and computational results were used to guide the design of an electromagnetic chicane for an LPA-driven XFEL experiment, enabling weight, size, and cost savings.

Future work includes the extension of the spline method to higher point counts. However, due to the exponential computational cost and diminishing returns, in addition to the complexities of fabricating such surfaces, it is perhaps not the most profitable path. Another figure of merit might be considered; instead of optimizing the field only on the transverse axis, the higher order multipole coefficients could be minimized. Another possible extension of the work is the inclusion of voids in the space above the pole face in order to tune the flux lines even before the iron-air interface, a la [24]. Finally, the modification of the side of the pole piece should be considered, especially in the context of very low AR magnets.

\section{ACKNOWLEDGMENTS}

This work was supported by the U.S. Department of Energy, Office of Science, Office of Workforce Development for Teachers and Scientists, Office of Science Graduate Student Research (SCGSR) program. The SCGSR program is administered by the Oak Ridge Institute for Science and Education for the DOE under Contract No. DE-SC0014664. This work was also supported by U.S. DOE Contracts No. DE-SC0009914 and No. DE-AC02-05CH11231 and by the Gordon and Betty Moore Foundation under Grant No. GBMF4898. The authors also wish to thank R. Robles and W. Lynn for their assistance with construction and testing of the UCLA-LBNL dipoles.

\section{APPENDIX A: GFQ $=10^{3}$}

The pole face is shown in gray, the field error is given as a density plot, the region satisfying GFQ is outlined in black, and the 0.9 gap unit radius geometric constraint is shown with the dashed line. Axes are labeled in gap units. Optimized pole faces and their equations for $\mathrm{GFQ}=10^{3}$ cases are shown in Figs. 12-26.

\section{1. $\mathrm{AR}=0.5$}

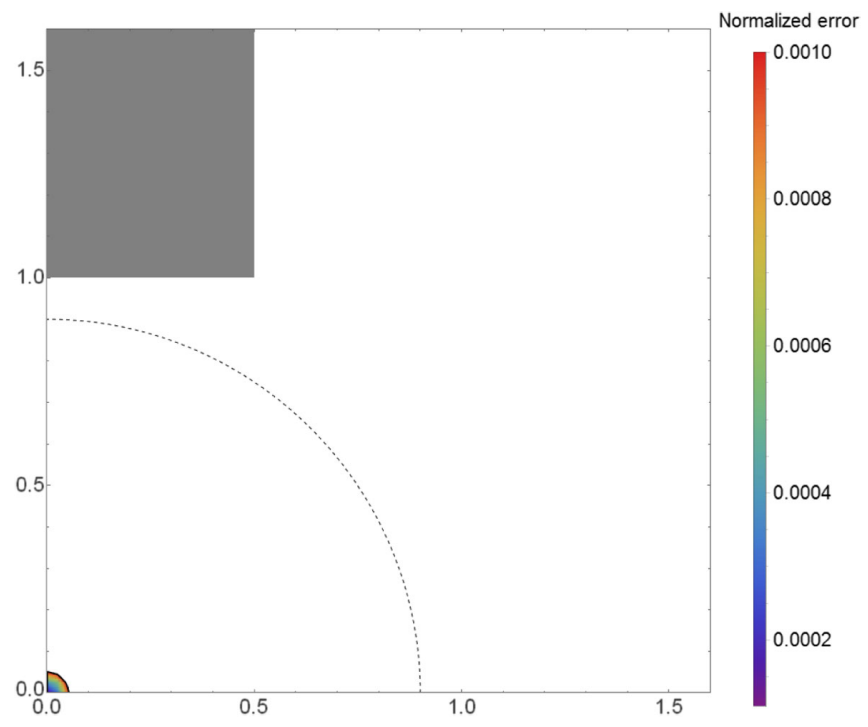

FIG. 12. Flat. $x_{\text {gfr }}=0.05$ gap units. No coefficients. 

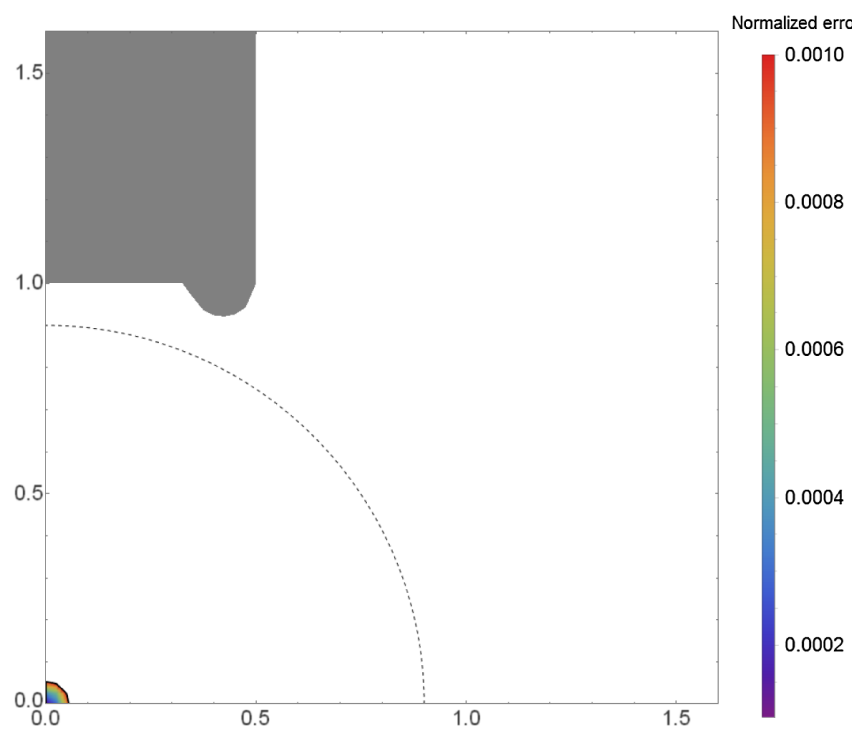

FIG. 13. Shimmed. $\mathrm{x}_{\mathrm{gfr}}=0.05$ gap units. Shim radius $=0.079$ gap units.

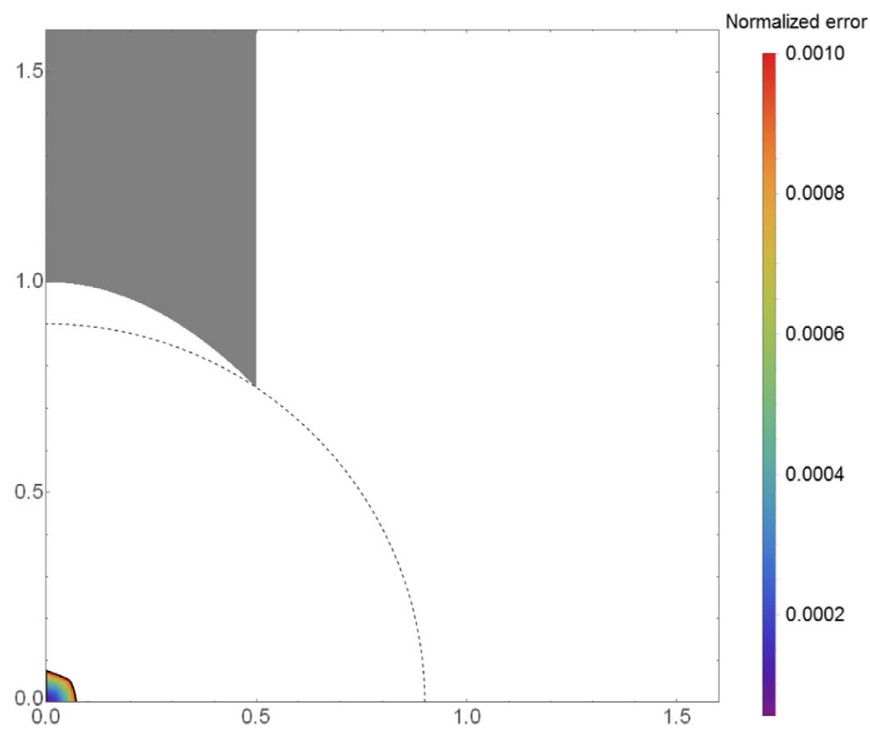

FIG. 14. Parabolic. $\mathrm{x}_{\text {gfr }}=0.07$ gap units. $\mathrm{y}=1-1.006 \mathrm{x}^{2}$.

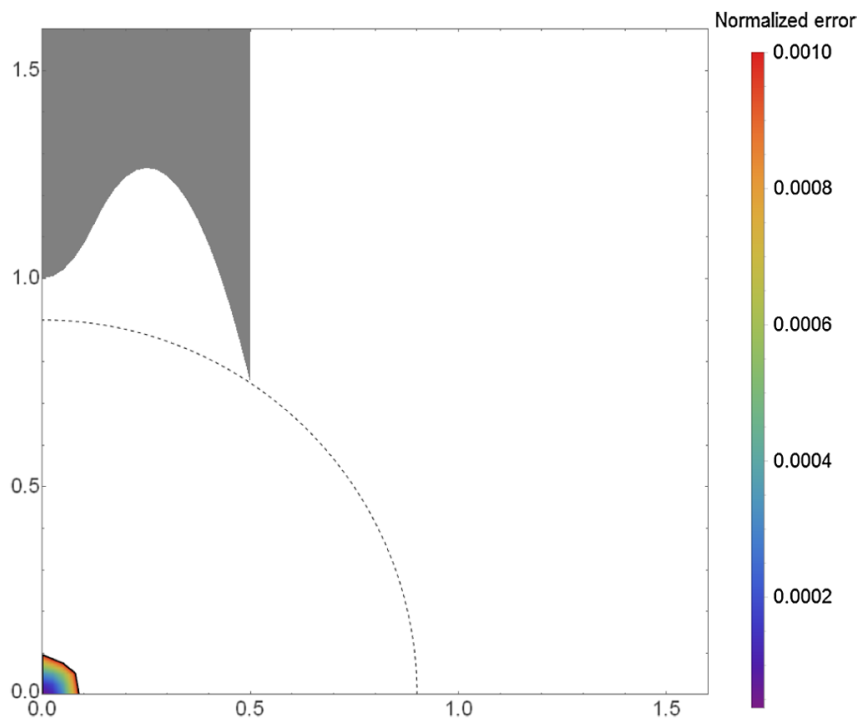

FIG. 15. 3 point spline. $\mathrm{x}_{\mathrm{gfr}}=0.09$ gap units. Spline points: $(0,1),(0.25,1.27),(0.5,0.75)$.

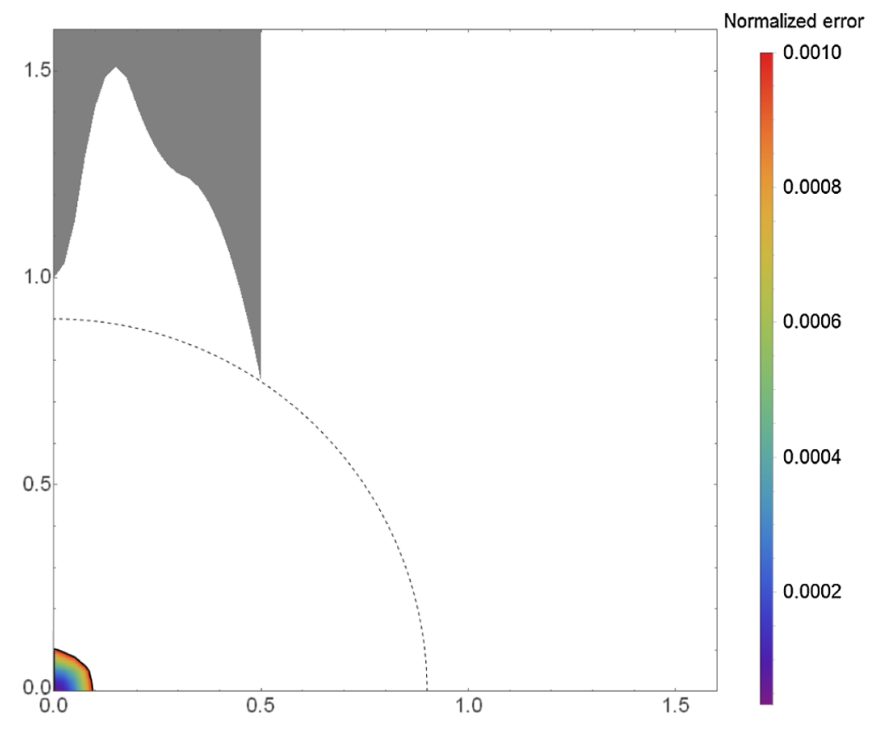

FIG. 16. 5 point spline. $\mathrm{x}_{\mathrm{gfr}}=0.09$ gap units. Spline points: $(0,1),(0.125,1.49),(0.25,1.31),(0.375,1.18),(0.5,0.75)$. 

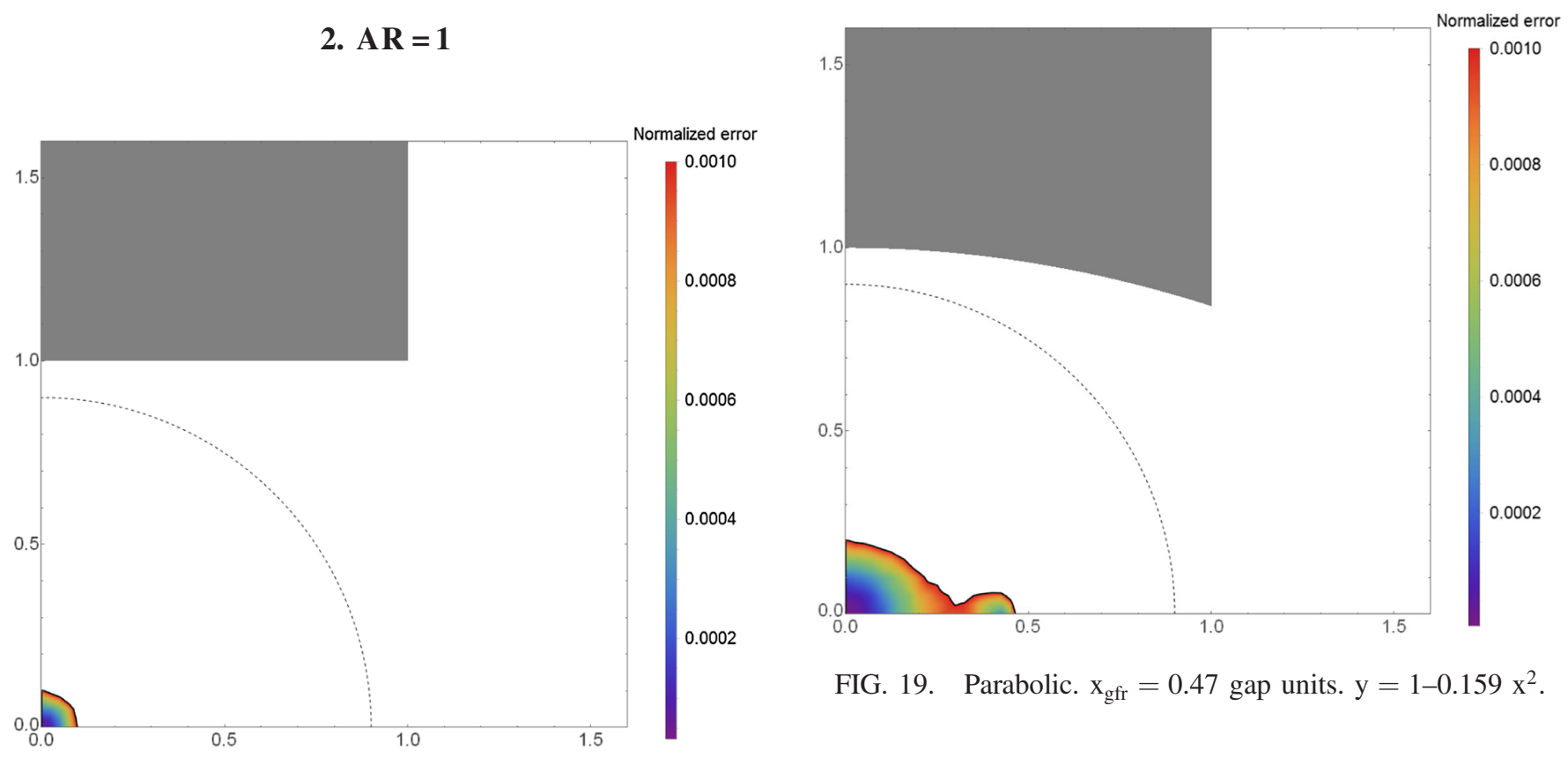

FIG. 19. Parabolic. $\mathrm{x}_{\mathrm{gfr}}=0.47$ gap units. $\mathrm{y}=1-0.159 \mathrm{x}^{2}$.

FIG. 17. Flat. $\mathrm{x}_{\mathrm{gfr}}=0.09$ gap units. No coefficients.

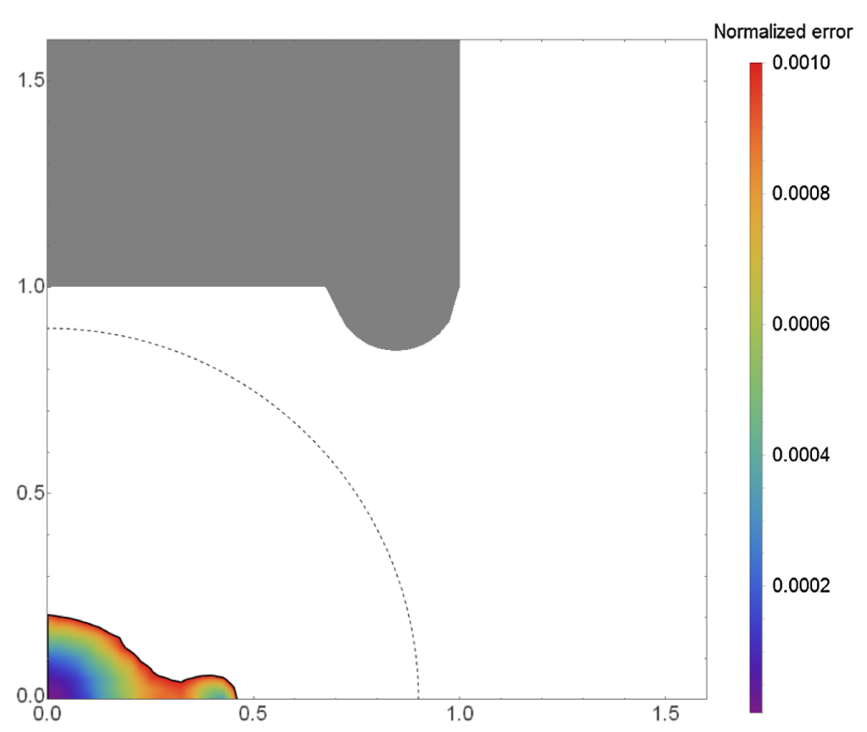

FIG. 18. Shimmed. $\mathrm{x}_{\mathrm{gfr}}=0.46$ gap units. Shim radius $=0.154$ gap units.

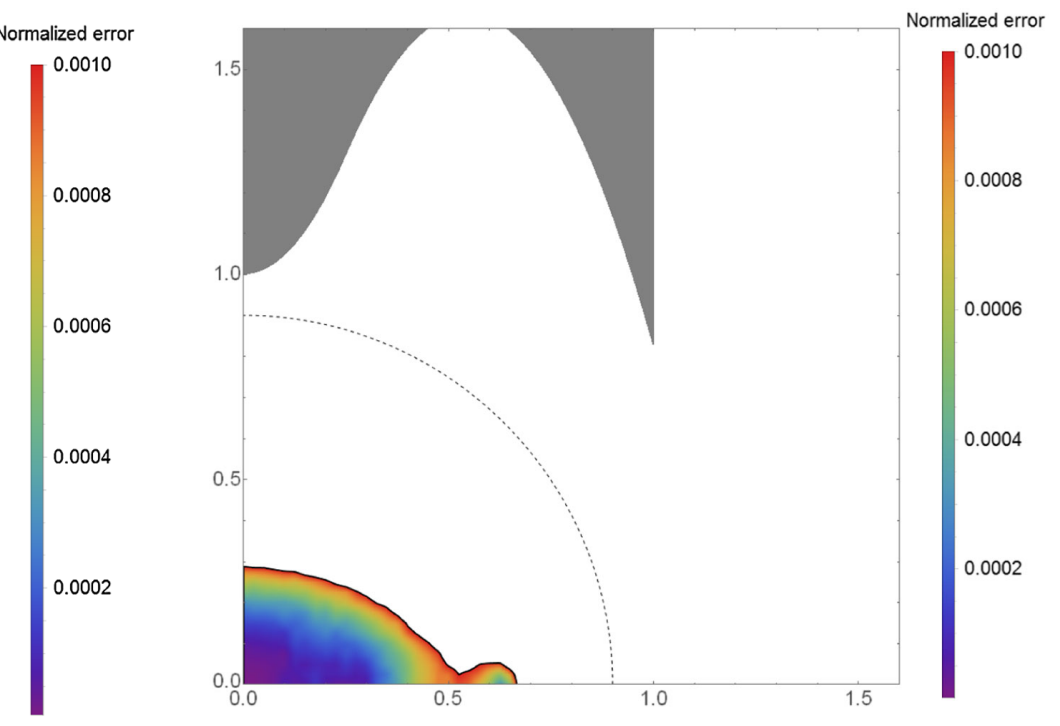

FIG. 20. 3 point spline. $\mathrm{x}_{\mathrm{gfr}}=0.67$ gap units. Spline points: $(0,1),(0.5,1.63),(1 ., 0.82)$. 


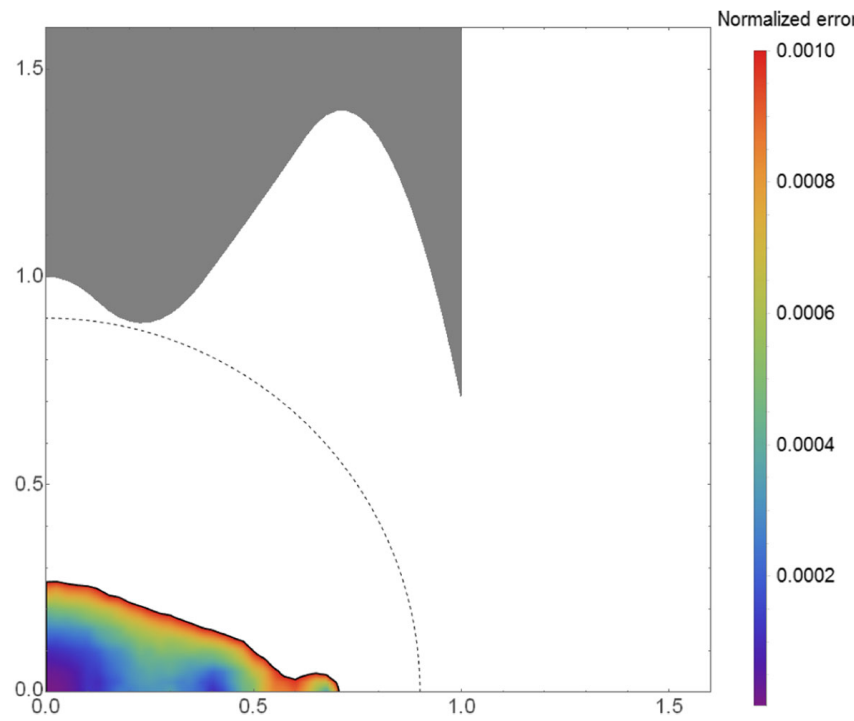

FIG. 21. 5 point spline. $\mathrm{x}_{\mathrm{gfr}}=0.71$ gap units. Spline points: $(0,1),(0.25,0.89),(0.5,1.16),(0.75,1.39),(1 ., 0.70)$.

\section{3. $\mathrm{AR}=1.5$}

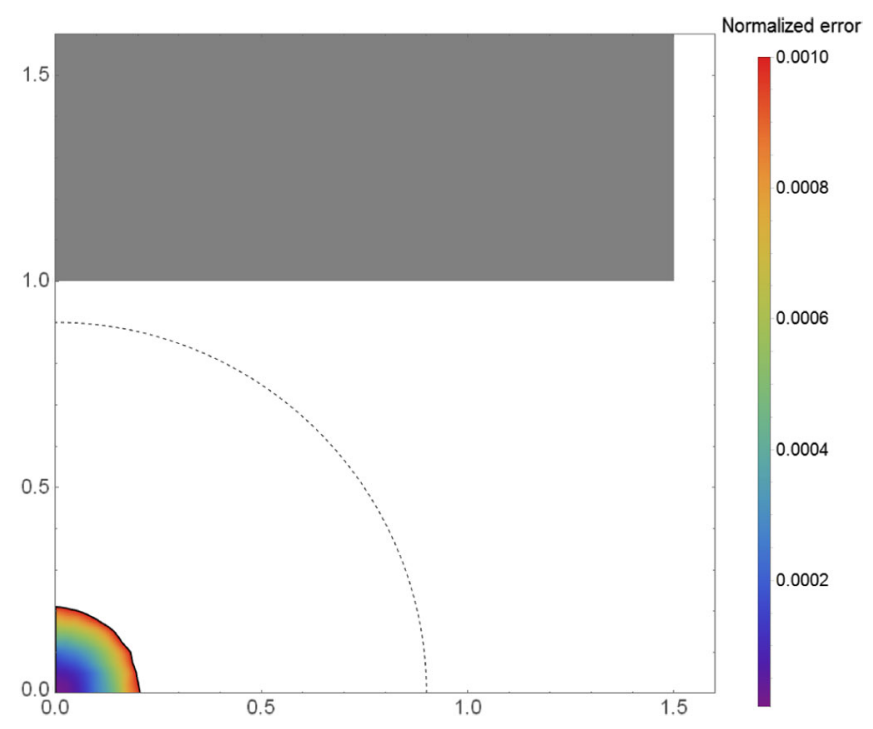

FIG. 22. Flat. $x_{\text {gfr }}=0.21$ gap units. No coefficients.

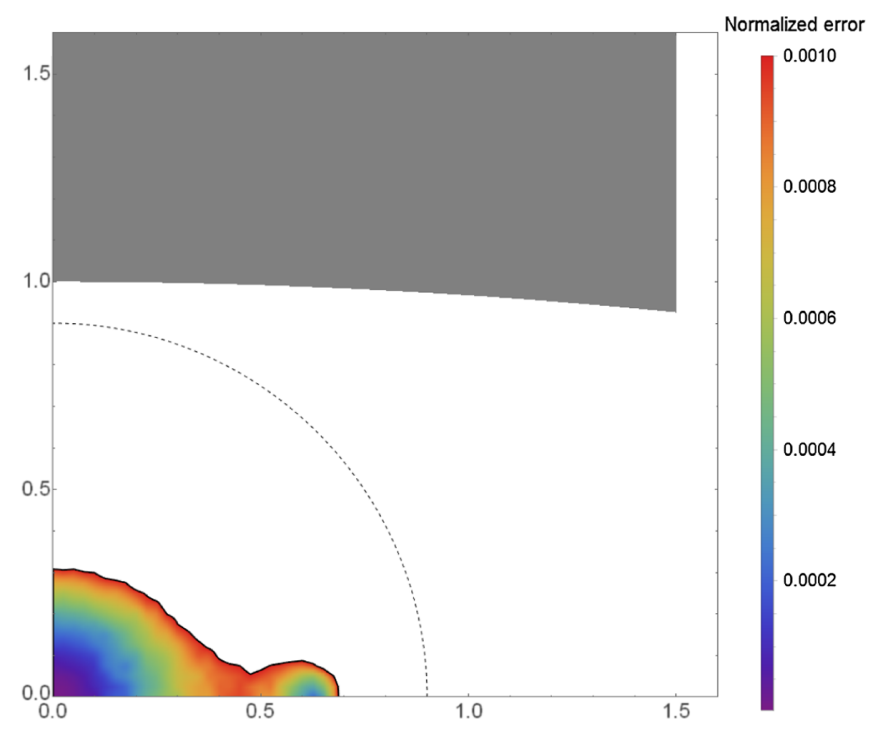

FIG. 24. Parabolic. $\mathrm{x}_{\mathrm{gfr}}=0.69$ gap units. $\mathrm{y}=1-0.033 \mathrm{x}^{2}$. 


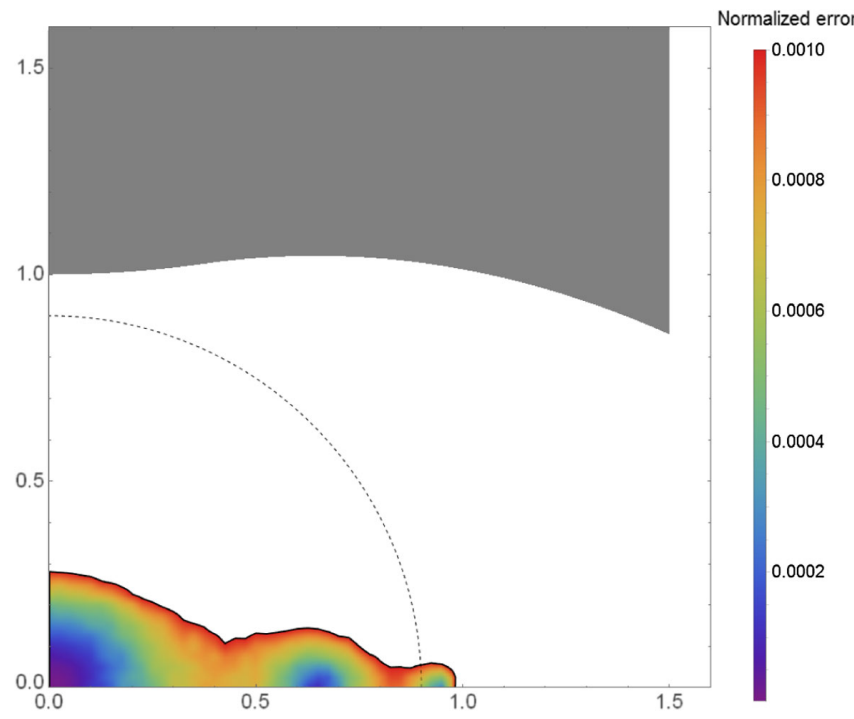

FIG. 25 . 3 point spline. $\mathrm{x}_{\mathrm{gfr}}=1.00$ gap units. Spline points: $(0,1),(0.75,1.04),(1.5,0.86)$.

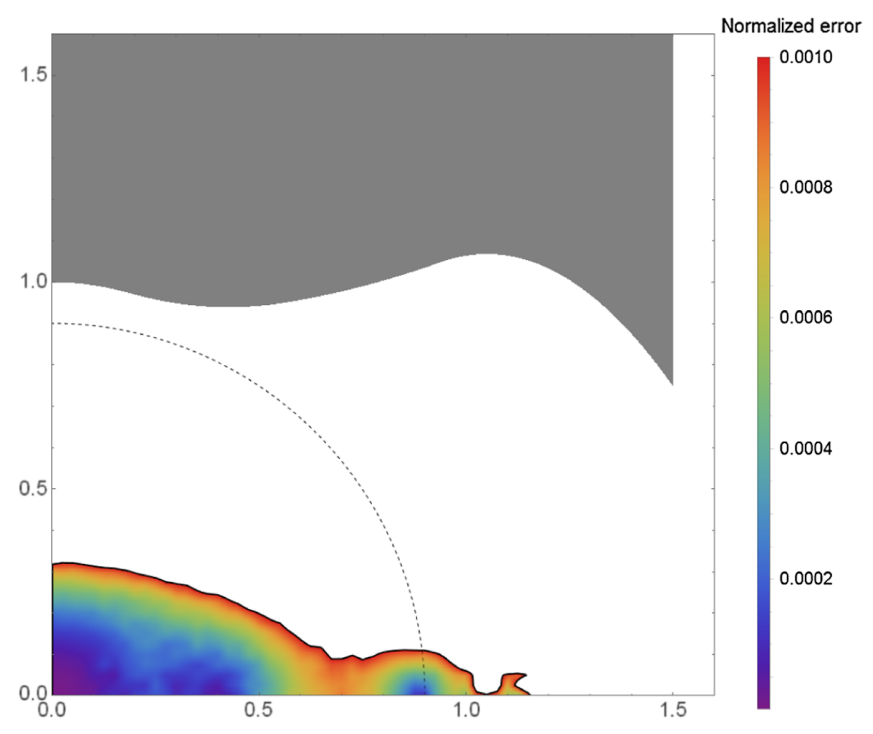

FIG. 26. 5 point spline. $\mathrm{x}_{\mathrm{gfr}}=1.17$ gap units. Spline points: $(0,1),(0.375,0.94),(0.75,0.99),(1.125,1.06),(1.5,0.75)$.

\section{APPENDIX B: GFQ $=10^{3.5}$}

\section{1. $\mathrm{AR}=0.5$}

Optimized pole faces and their equations for $\mathrm{GFQ}=10^{3.5}$ cases are shown in Figs. 27-41.

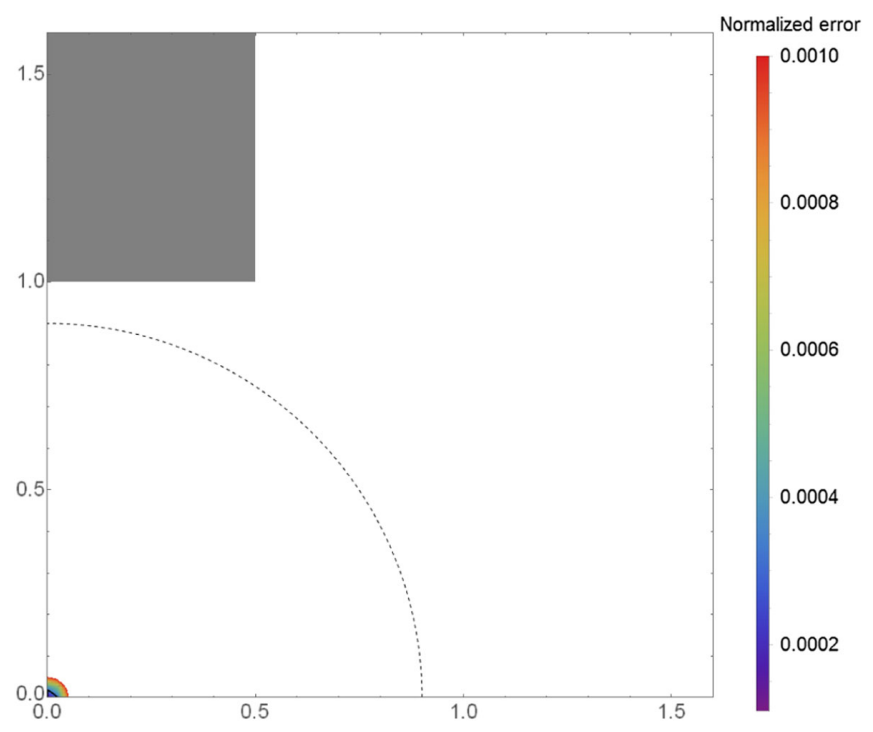

FIG. 27. Flat. $\mathrm{x}_{\mathrm{gfr}}=0.03$ gap units. No coefficients.

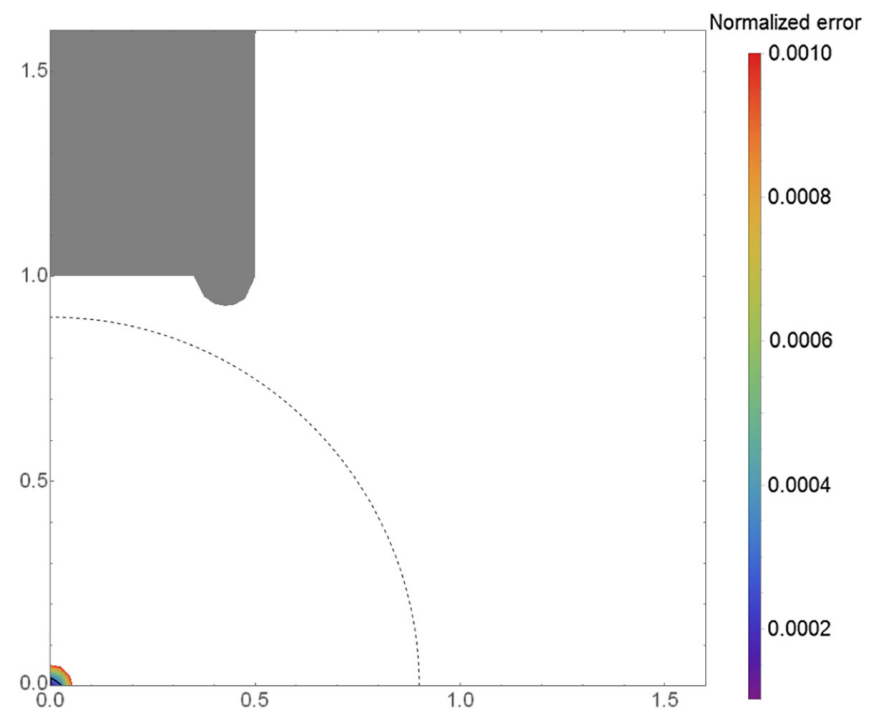

FIG. 28. Shimmed. $\mathrm{x}_{\mathrm{gfr}}=0.03$ gap units. Shim radius $=0.072$ gap units. 


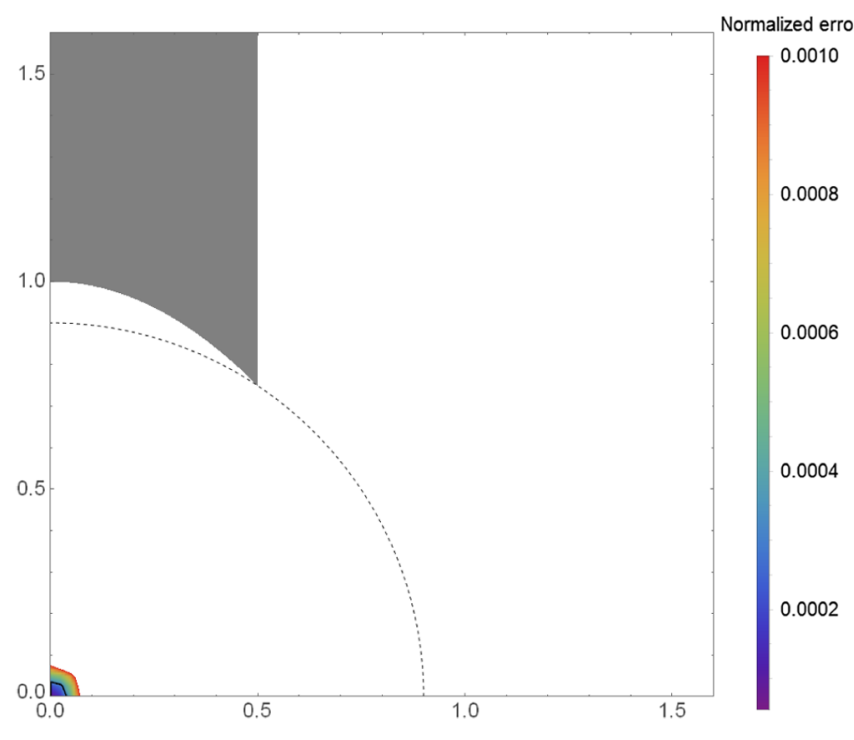

FIG. 29. Parabolic. $\mathrm{x}_{\mathrm{gfr}}=0.04$ gap units. $\mathrm{y}=1-1.006 \mathrm{x}^{2}$.

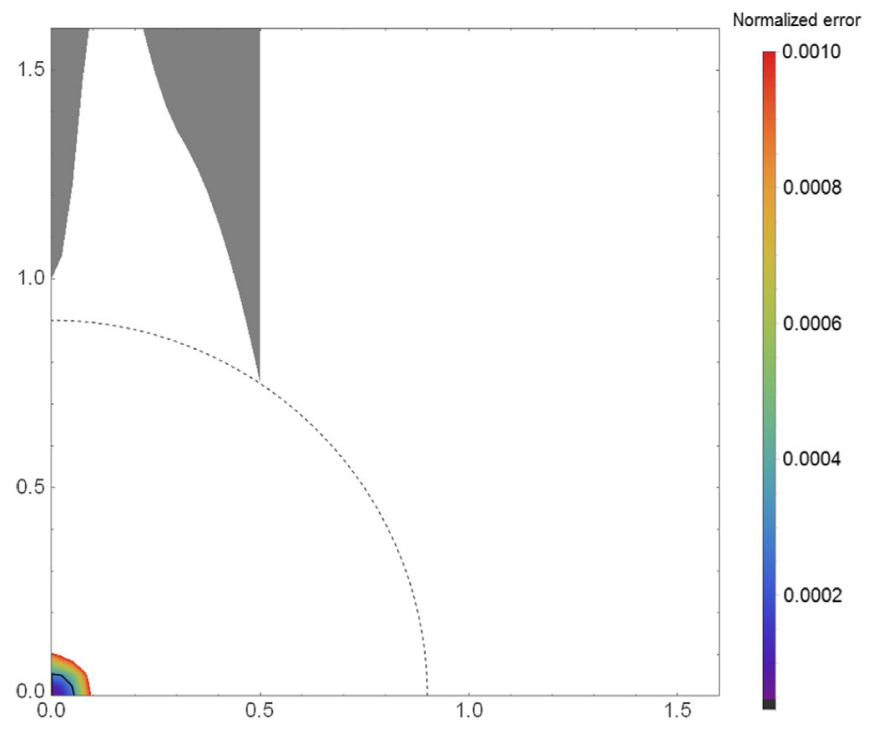

FIG. 31 . 5 point spline. $\mathrm{x}_{\mathrm{gfr}}=0.05$ gap units. Spline points: $(0,1),(0.125,1.80),(0.25,1.48),(0.375,1.20),(0.5,0.75)$.

\section{2. $\mathbf{A} \mathbf{R}=\mathbf{1}$}

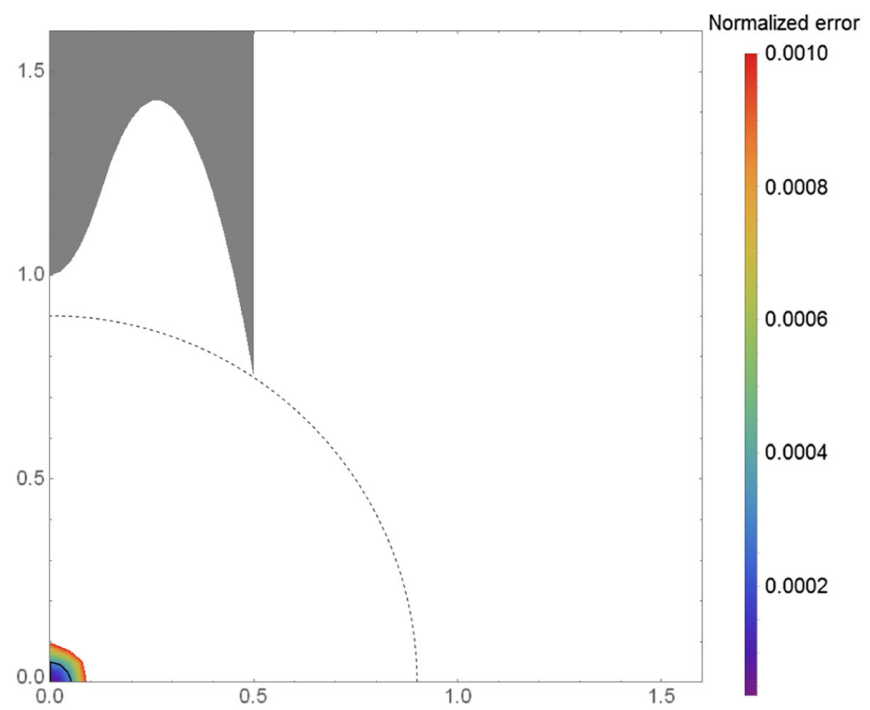

FIG. 30.3 point spline. $x_{\text {gfr }}=0.05$ gap units. Spline points: $(0,1),(0.25,1.43),(0.5,0.75)$.

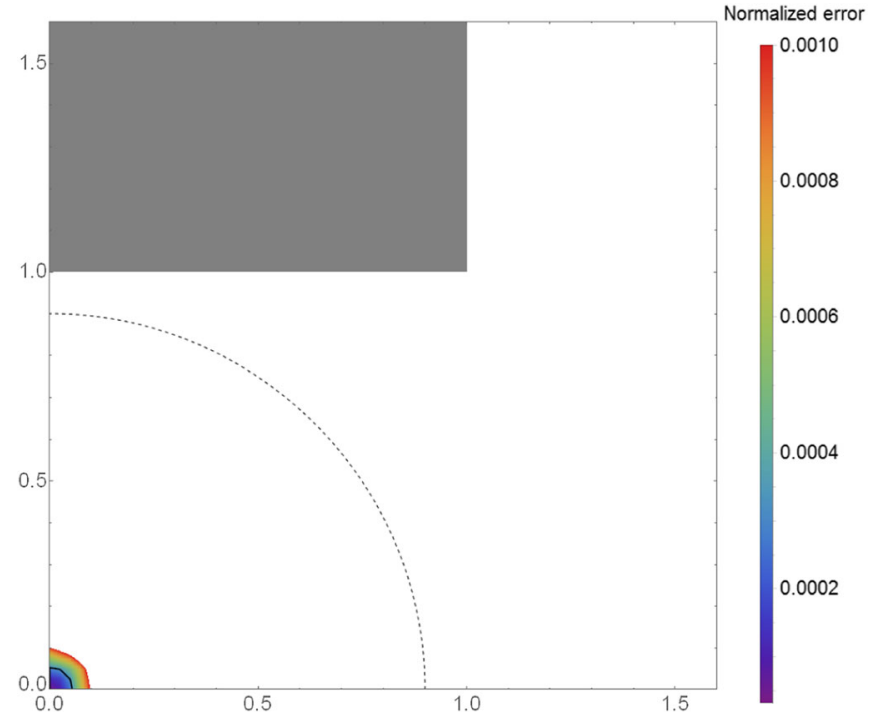

FIG. 32. Flat. $\mathrm{x}_{\mathrm{gfr}}=0.05$ gap units. No coefficients. 


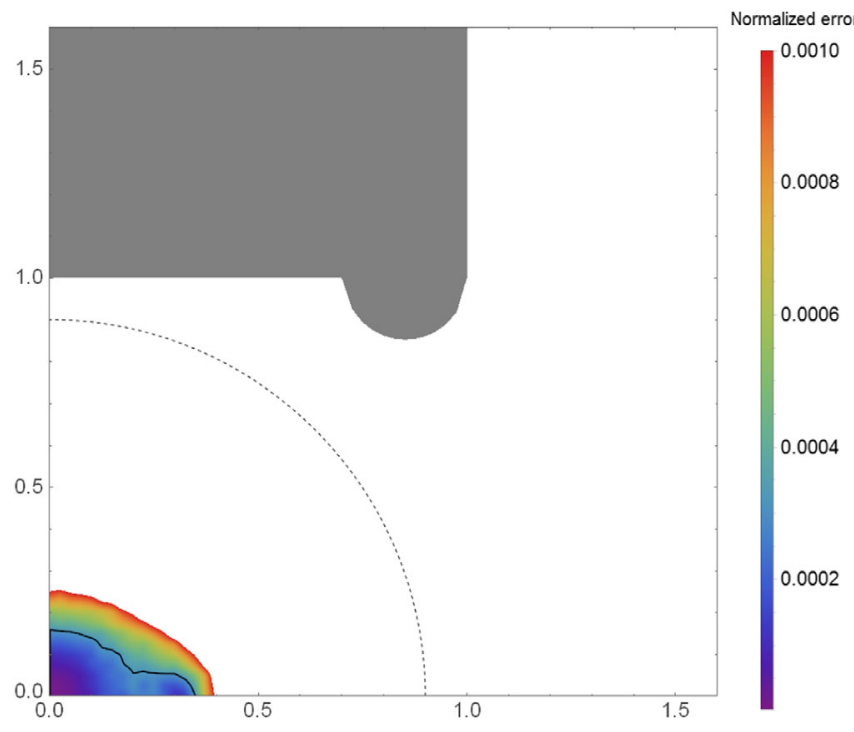

FIG. 33. Shimmed. $\mathrm{x}_{\mathrm{gfr}}=0.35$ gap units. Shim radius $=0.148$ gap units.

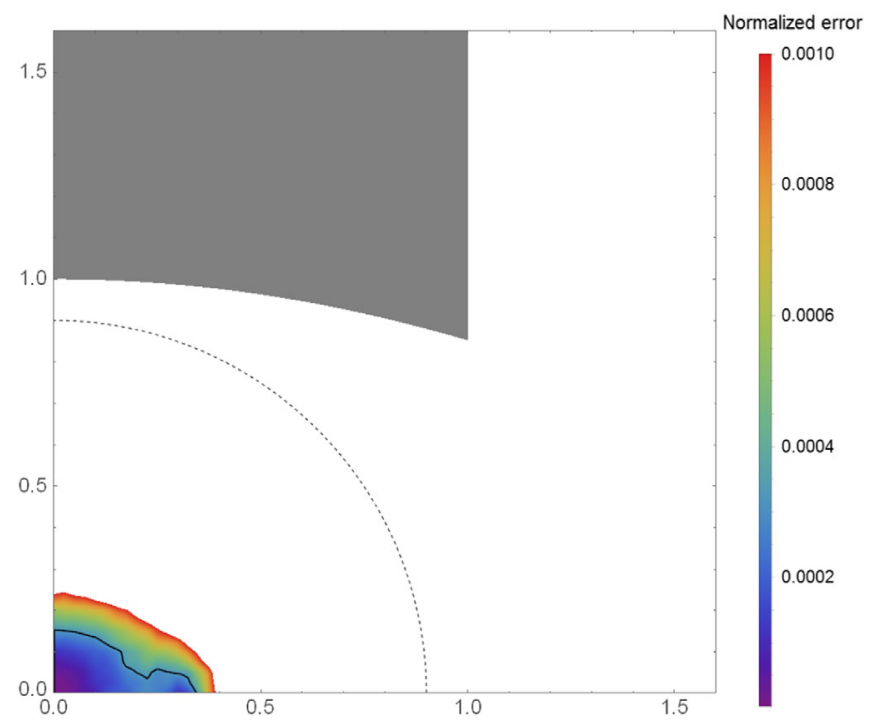

FIG. 34. Parabolic. $\mathrm{x}_{\mathrm{gfr}}=0.35$ gap units. $y=1-0.148 \mathrm{x}^{2}$.

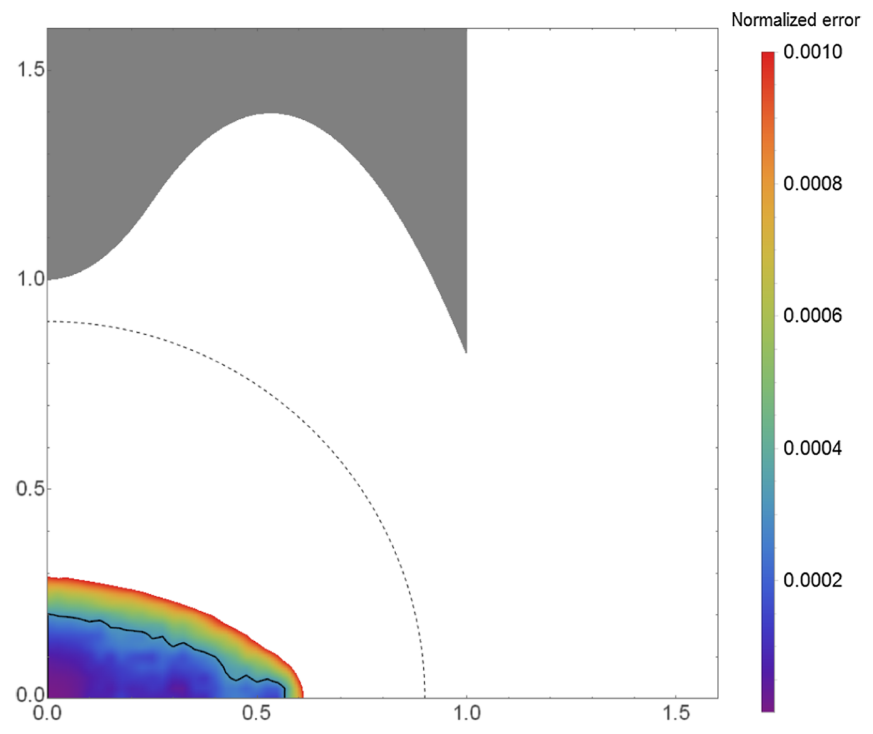

FIG. 35. 3 point spline. $\mathrm{x}_{\mathrm{gfr}}=0.57$ gap units. Spline points: $(0,1),(0.5,1.39),(1 ., 0.82)$.

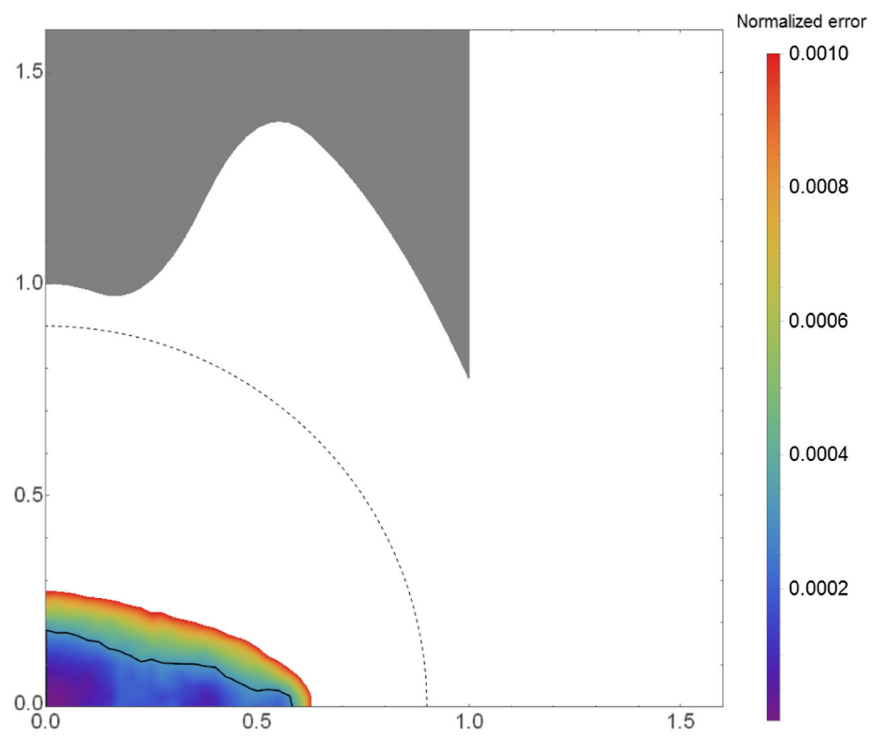

FIG. 36. 5 point spline. $\mathrm{x}_{\mathrm{gfr}}=0.59$ gap units. Spline points: $(0,1),(0.25,1.01),(0.5,1.37),(0.75,1.21),(1 ., 0.77)$. 

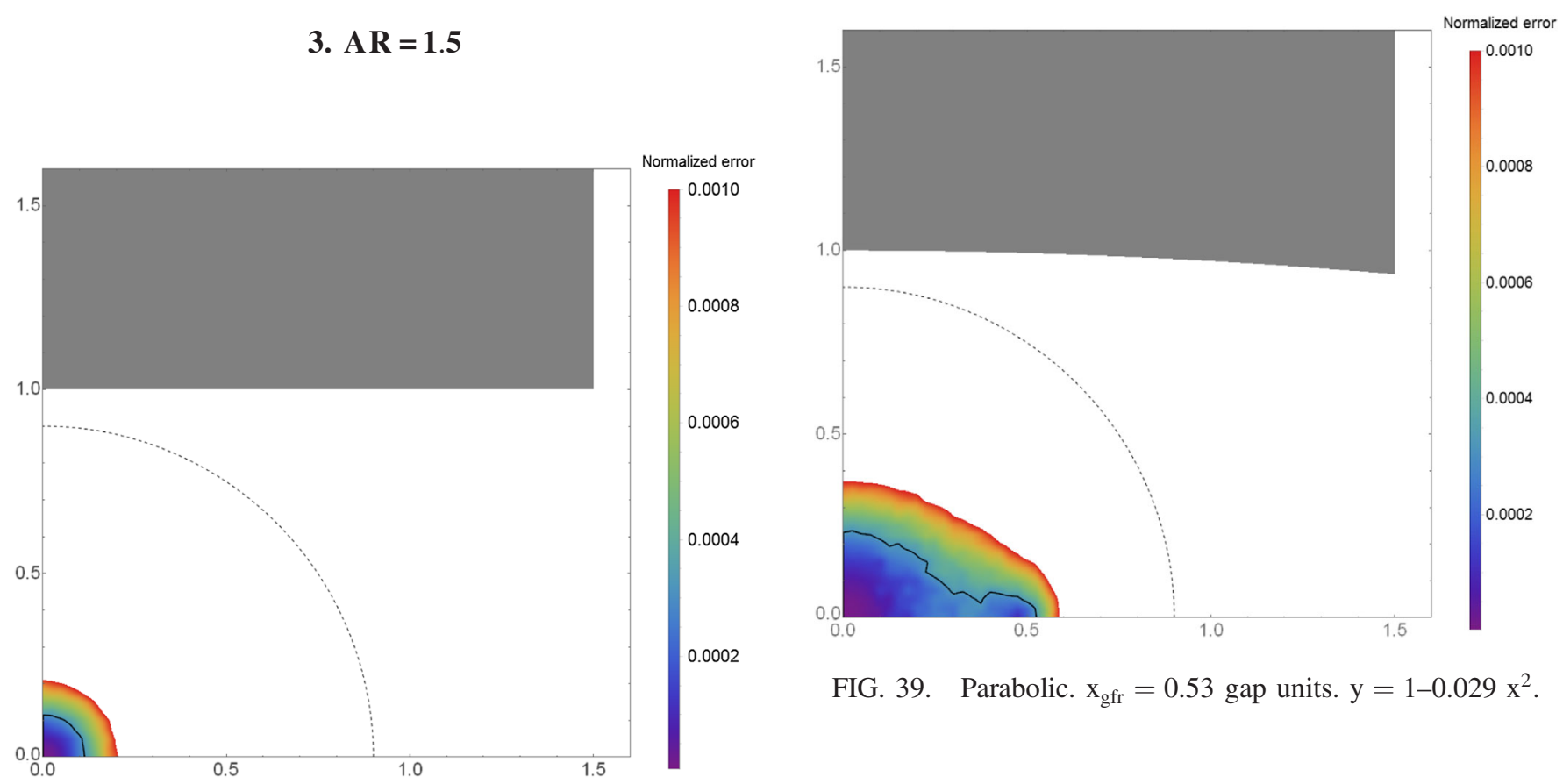

FIG. 39. Parabolic. $x_{\text {gfr }}=0.53$ gap units. $y=1-0.029 x^{2}$.

FIG. 37. Flat. $\mathrm{x}_{\mathrm{gfr}}=0.12$ gap units. No coefficients.

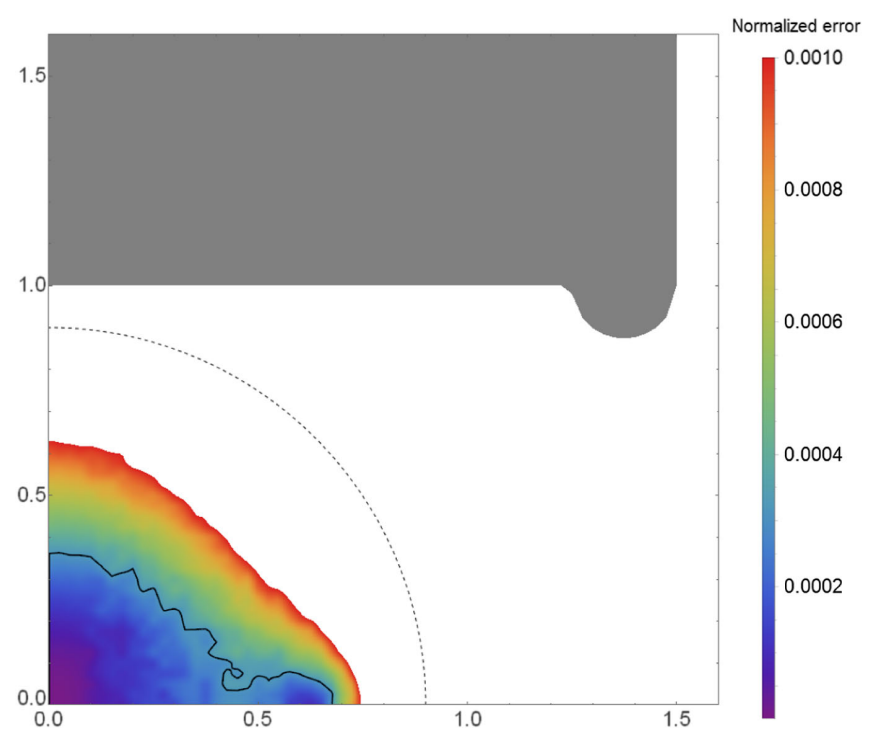

FIG. 38. Shimmed. $\mathrm{x}_{\mathrm{gfr}}=0.68$ gap units. Shim radius $=0.126$ gap units.

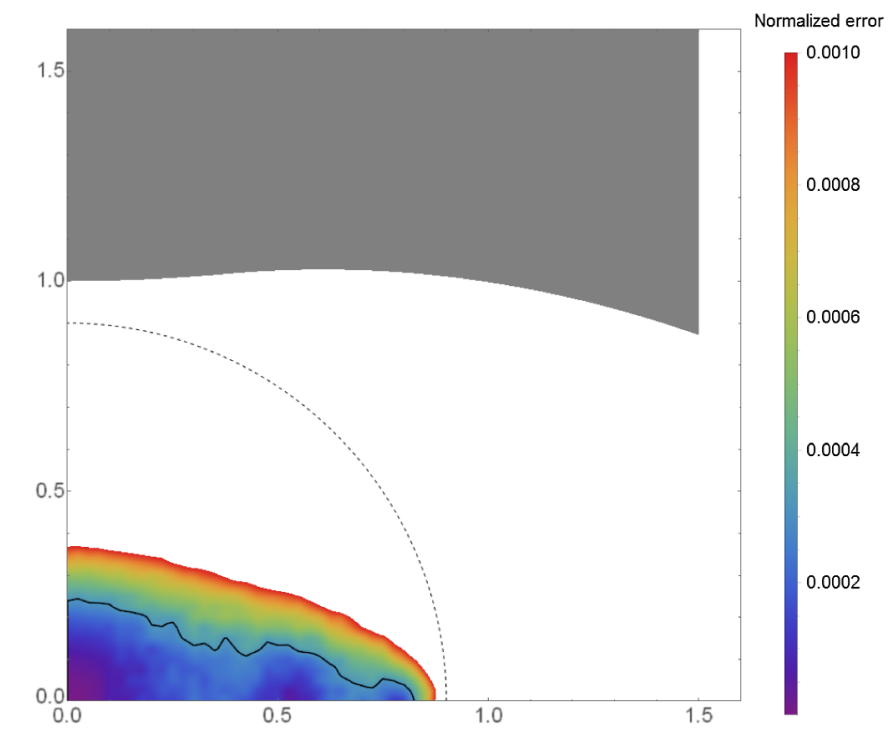

FIG. 40 . 3 point spline. $\mathrm{x}_{\mathrm{gfr}}=0.83$ gap units. Spline points: $(0,1),(0.75,1.02),(1.5,0.87)$. 


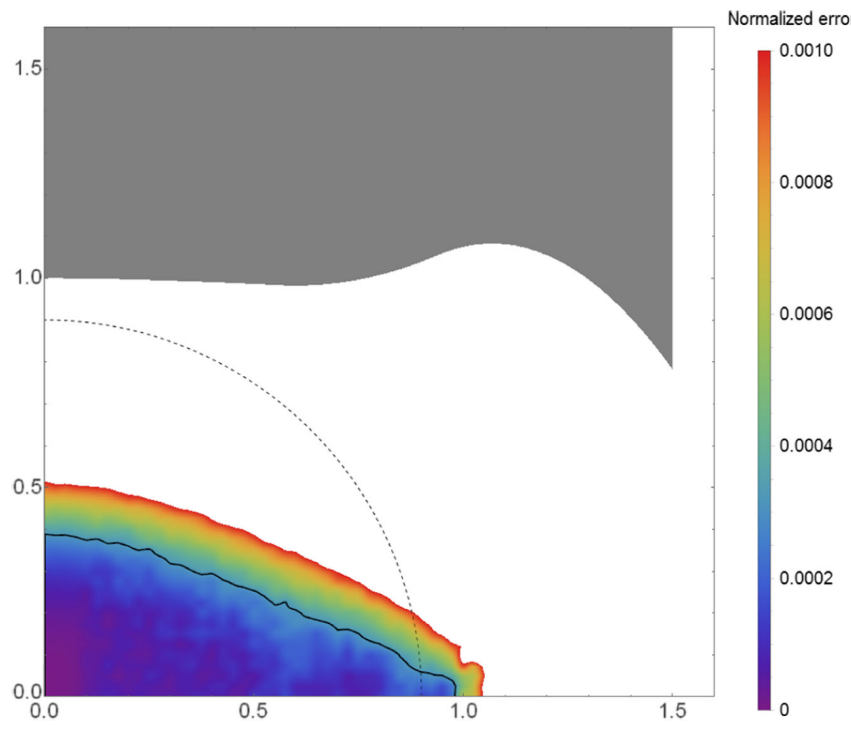

FIG. 41. 5 point spline. $\mathrm{x}_{\mathrm{gfr}}=1$ gap units. Spline points: $(0,1)$, $(0.375,0.99),(0.75,1.00),(1.125,1.08),(1.5,0.78)$.

\section{APPENDIX C: $\mathrm{GFQ}=10^{4}$}

\section{1. $\mathrm{AR}=0.5$}

Optimized pole faces and their equations for GFQ $=10^{4}$ cases are shown in Figs. 42-56.

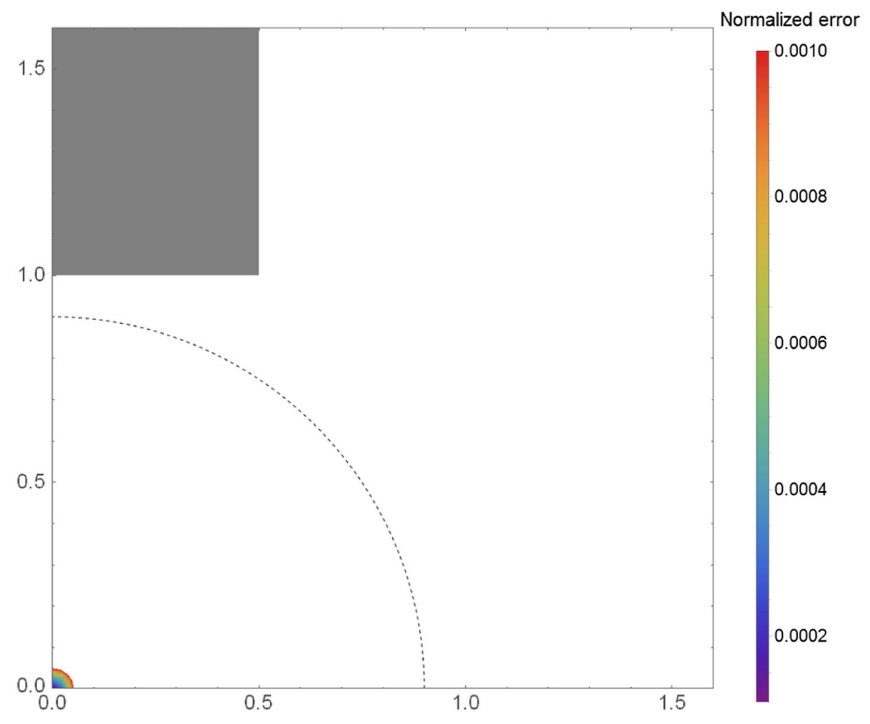

FIG. 42. Flat. $x_{\text {gfr }}=0.02$ gap units. No coefficients.

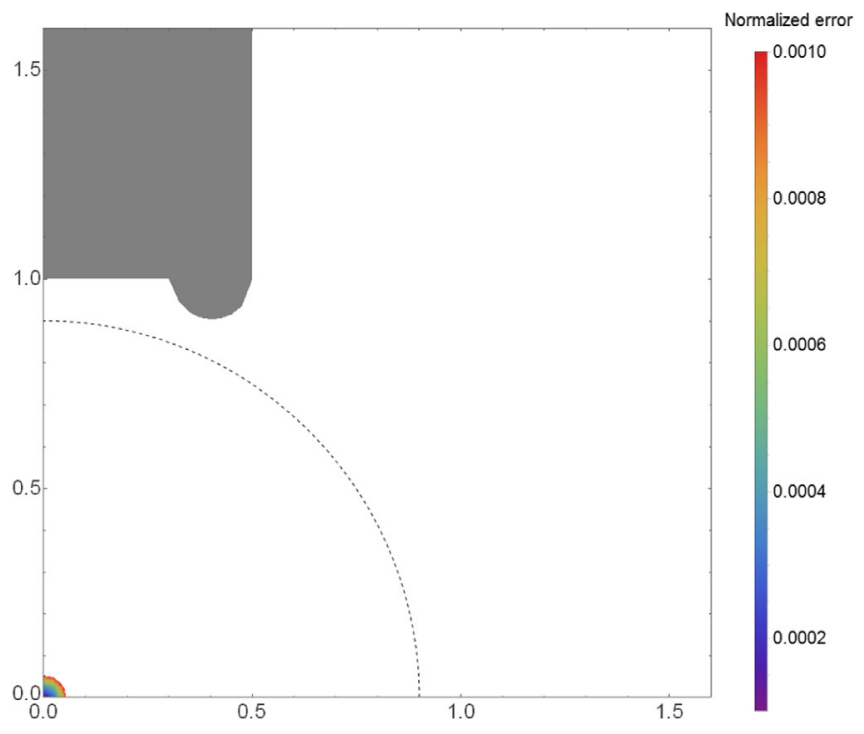

FIG. 43. Shimmed. $x_{\text {gfr }}=0.02$ gap units. Shim radius $=0.096$ gap units.

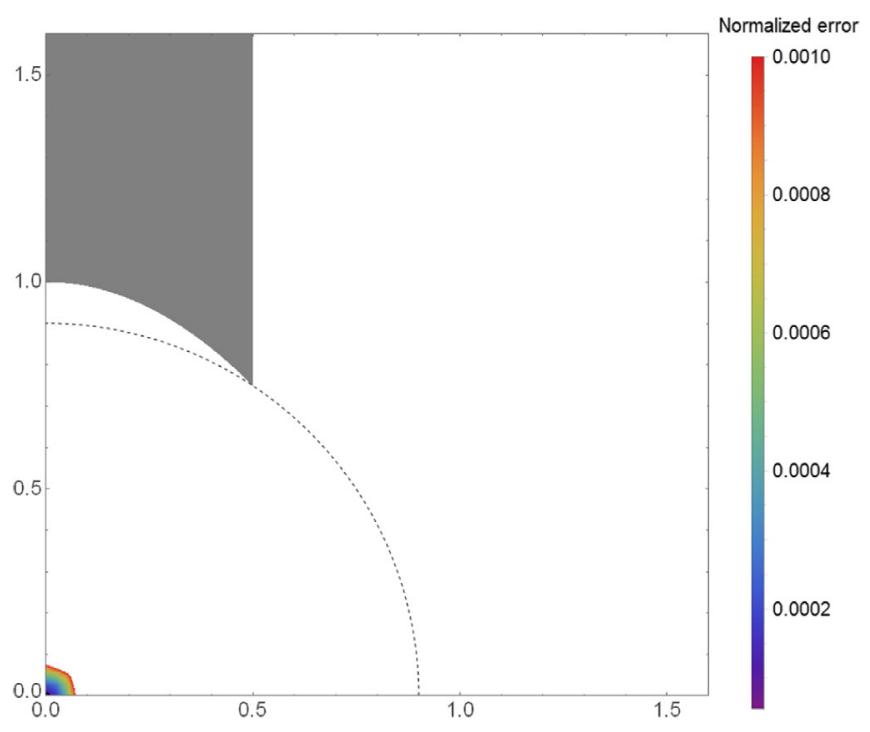

FIG. 44. Parabolic. $\mathrm{x}_{\mathrm{gfr}}=0.02$ gap units $y=1-1.006 \mathrm{x}^{2}$. 


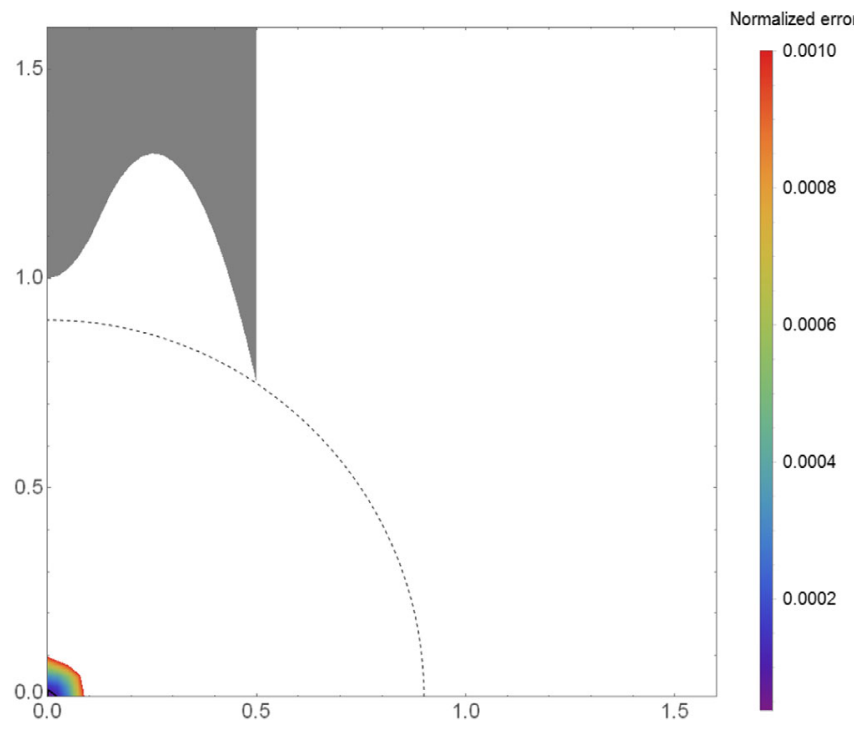

FIG. 45.3 point spline $\mathrm{x}_{\mathrm{gfr}}=0.03$ gap units. Spline points: $(0,1),(0.25,1.30),(0.5,0.75)$.

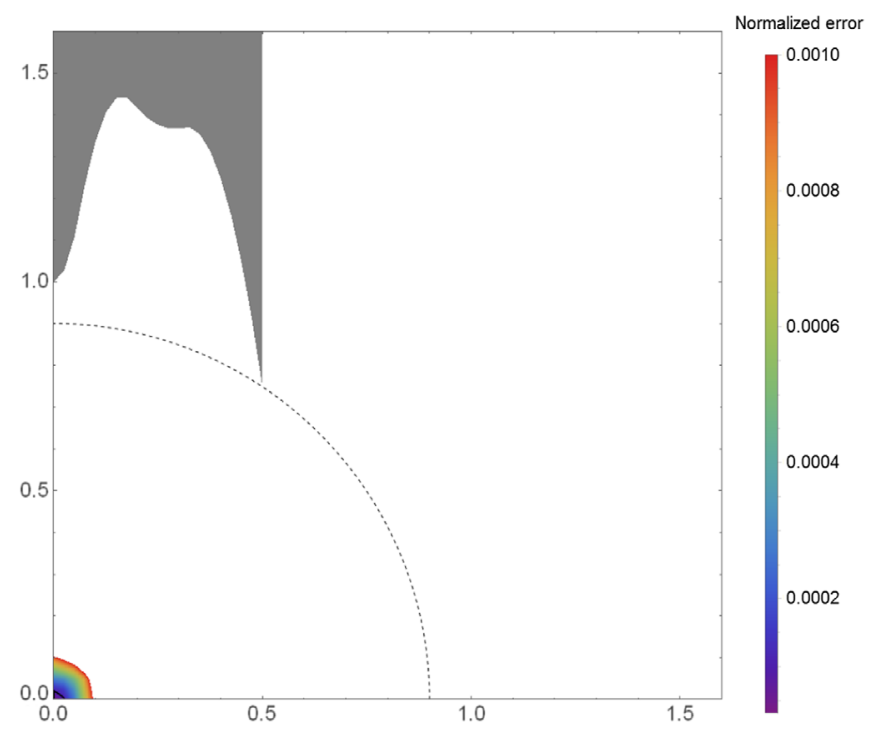

FIG. 46. 5 point spline. $\mathrm{x}_{\mathrm{gfr}}=0.03$ gap units. Spline points: $(0,1),(0.125,1.41),(0.25,1.38),(0.375,1.31),(0.5,0.75)$.

\section{2. $\mathbf{A R}=1$}

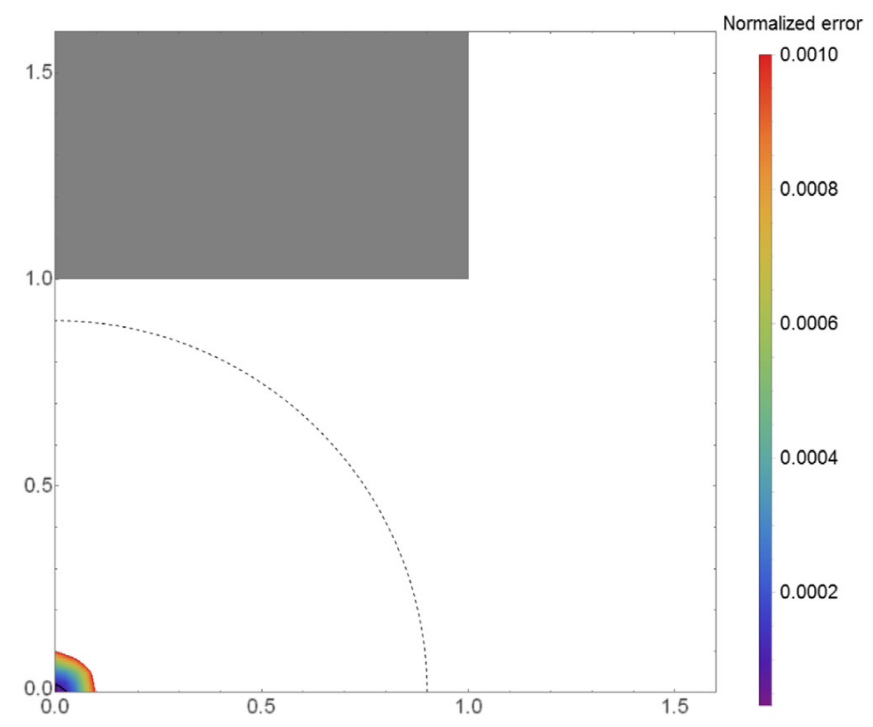

FIG. 47. Flat. $\mathrm{x}_{\text {gfr }}=0.03$ gap units. No coefficients.

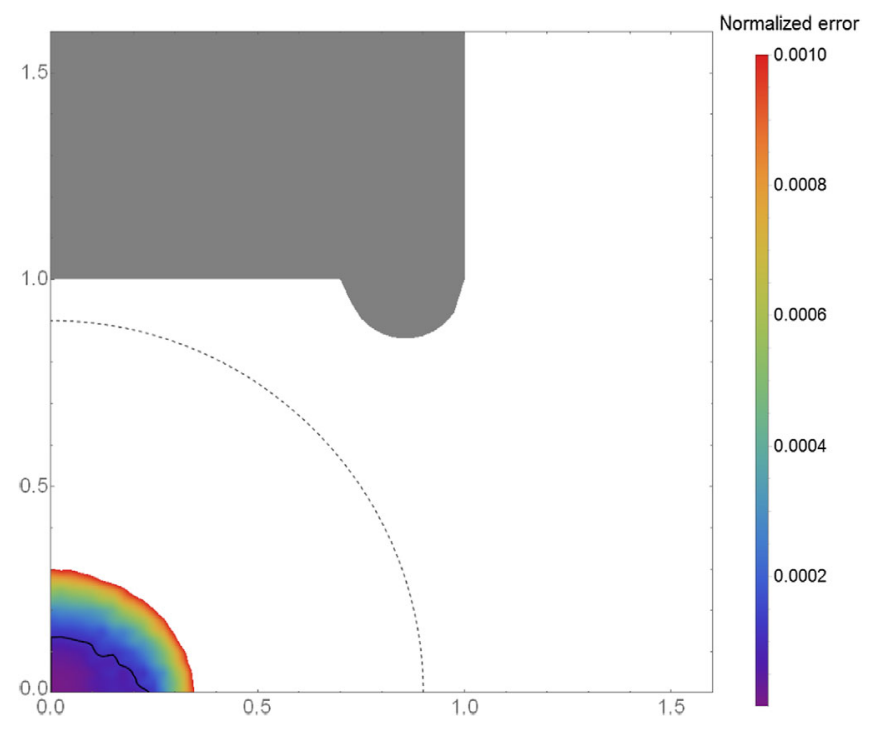

FIG. 48. Shimmed. $\mathrm{x}_{\mathrm{gfr}}=0.21$ gap units. Shim radius $=0.143$ gap units. 


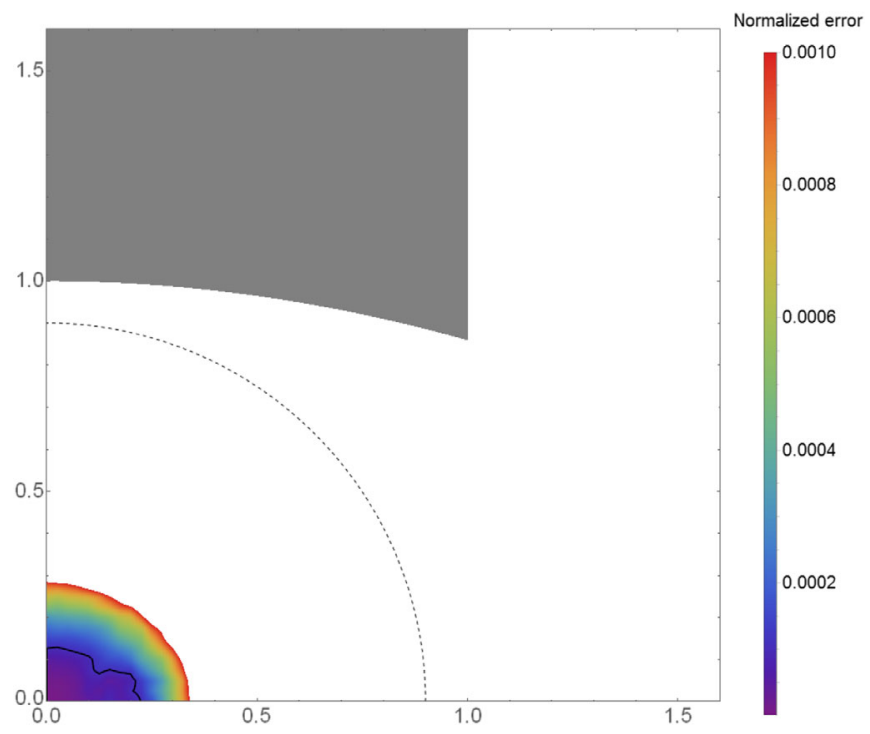

FIG. 49. Parabolic. $\mathrm{x}_{\mathrm{gfr}}=0.21$ gap units. $\mathrm{y}=1-0.141 \mathrm{x}^{2}$.

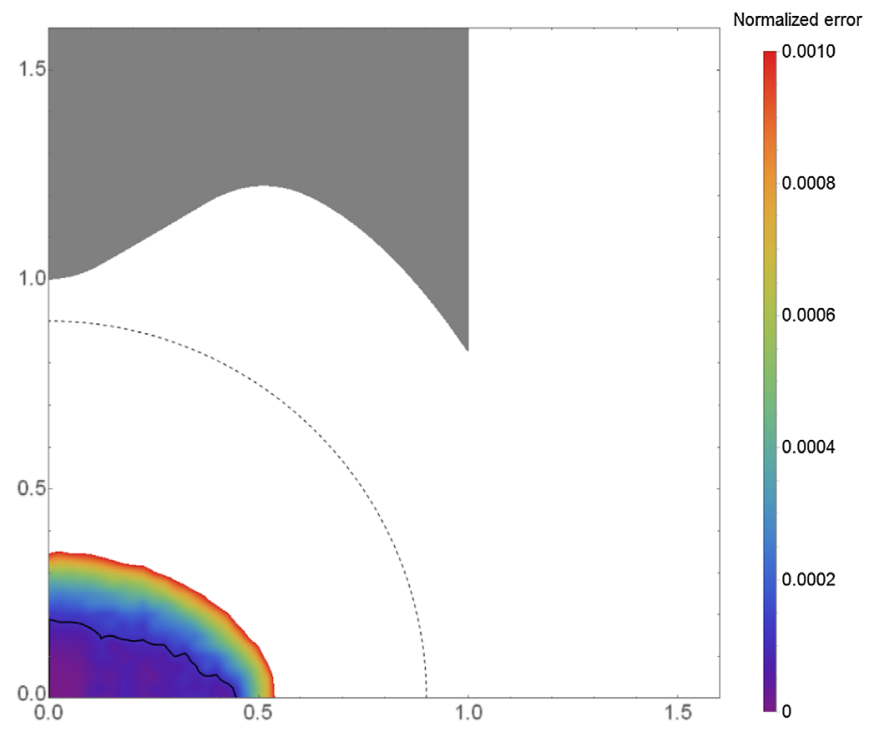

FIG. 51.5 point spline. $\mathrm{x}_{\mathrm{gfr}}=0.45$ gap units. Spline points: $(0,1),(0.25,1.11),(0.5,1.22),(0.75,1.11),(1 ., 0.82)$.

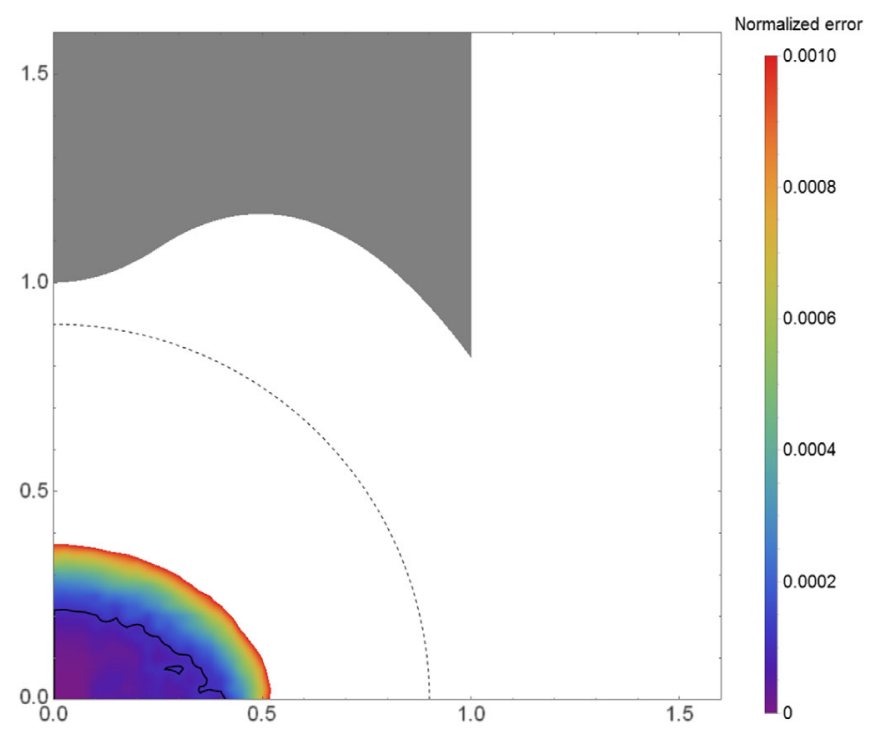

FIG. 50. 3 point spline. $\mathrm{x}_{\mathrm{gfr}}=0.41$ gap units. Spline points: $(0,1),(0.5,1.16),(1 ., 0.82)$.

\section{3. $\mathrm{AR}=1.5$}

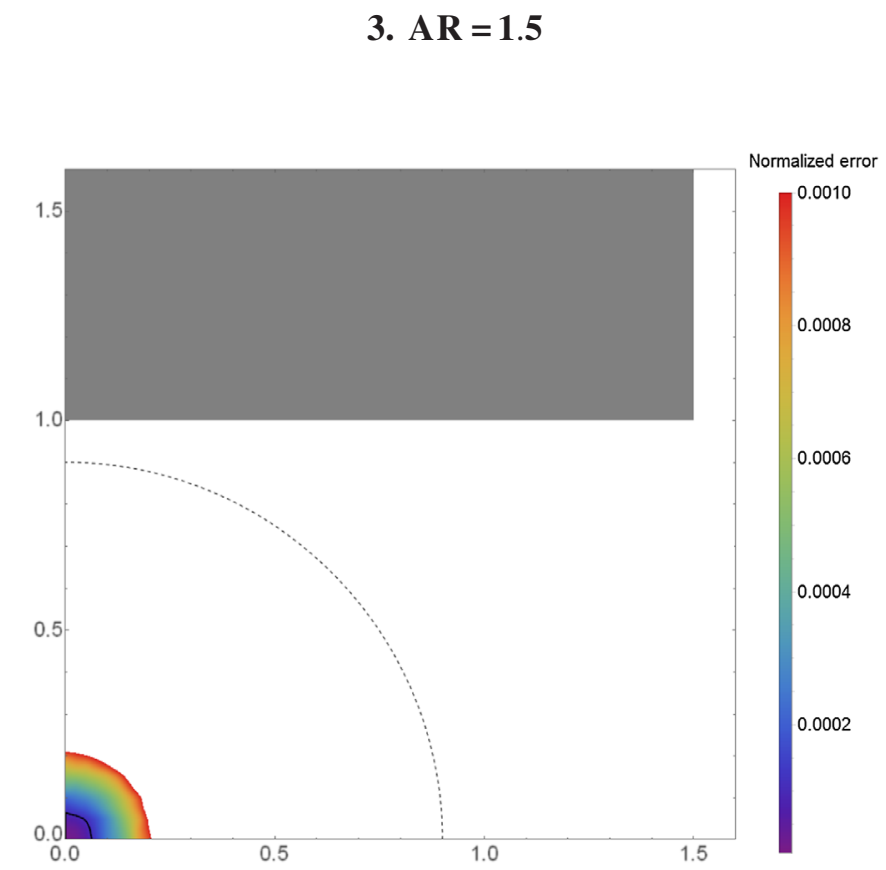

FIG. 52. Flat. $x_{\text {gfr }}=0.06$ gap units. No coefficients. 


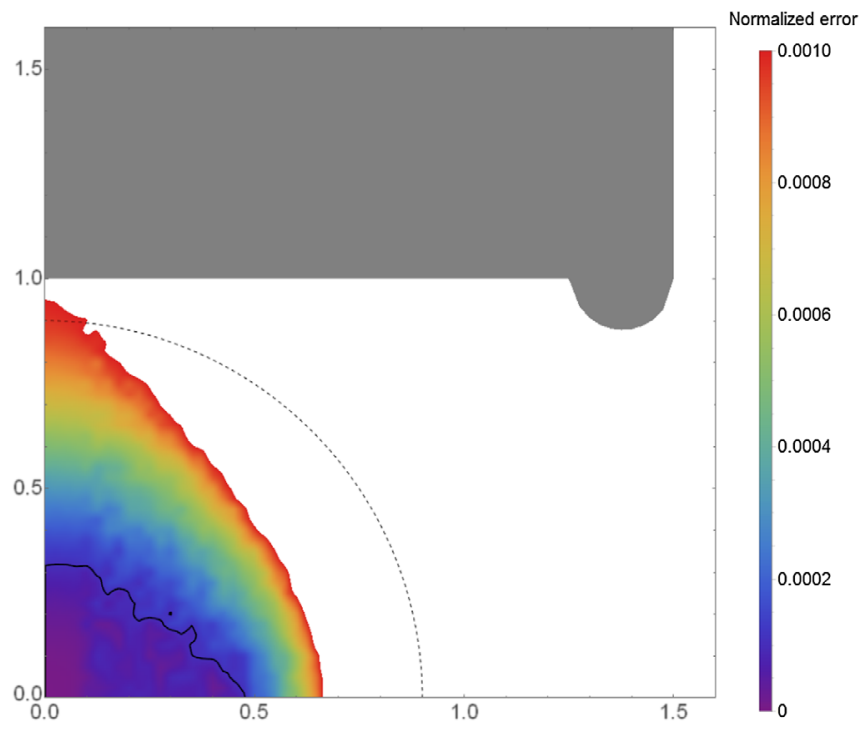

FIG. 53. Shimmed. $x_{\text {gfr }}=0.45$ gap units. Shim radius $=0.122$ gap units.

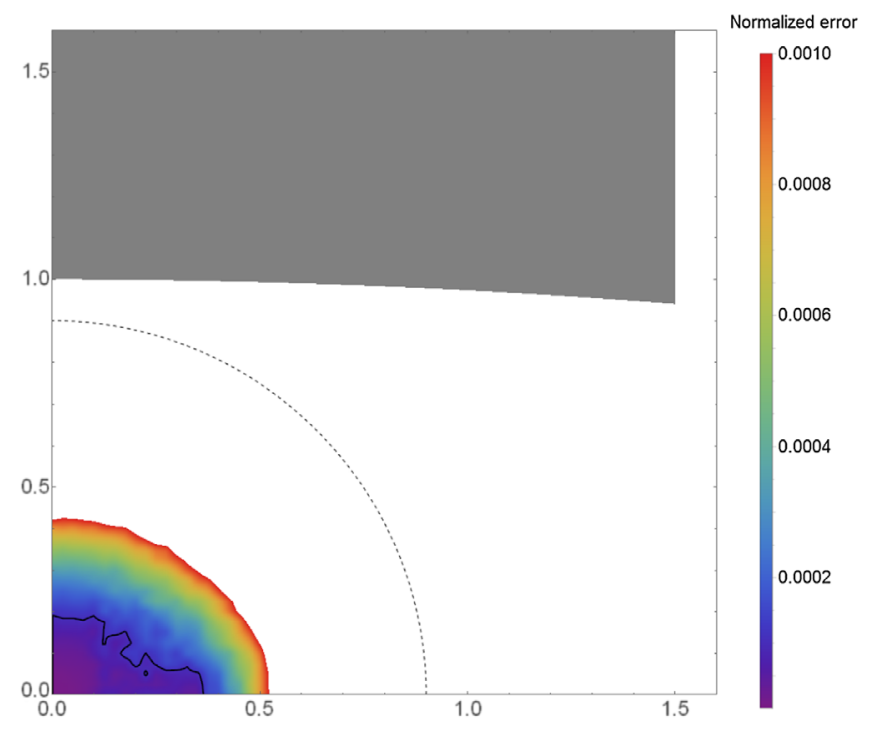

FIG. 54. Parabolic. $\mathrm{x}_{\mathrm{gfr}}=0.37$ gap units. $\mathrm{y}=1-0.026 \mathrm{x}^{2}$.

[1] J. Tanabe, Iron dominated electromagnets: Design, fabrication, assembly and measurements, Report No. SLAC-R-754, 2005.

[2] C. G. R. Geddes, Cs. Toth, J. van Tilborg, E. Esarey, C. B. Schroeder, D. Bruhwiler, C. Nieter, J. Cary, and W. P. Leemans, High-quality electron beams from a laser wakefield accelerator using plasma-channel guiding, Nature (London) 431, 538 (2004).

[3] S. Caspi, D. Arbelaez, L. Brouwer, S. Gourlay, S. Prestemon, and B. Auchmann, Design of a cantedcosine-theta superconducting dipole magnet for future colliders, IEEE Trans. Appl. Supercond. 27, 1 (2017).

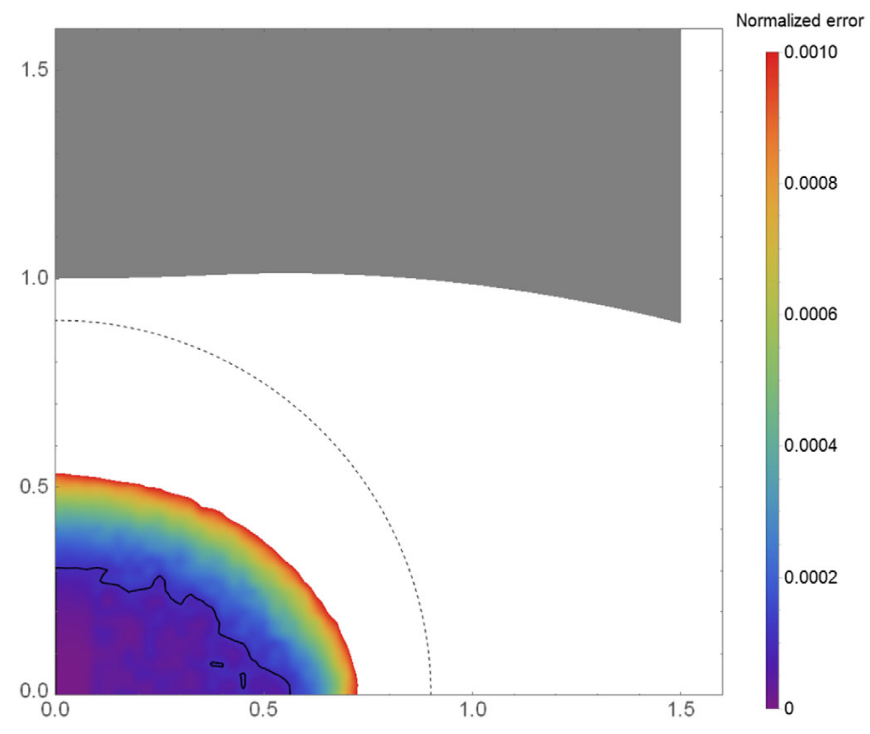

FIG. 55. 3 point spline. $\mathrm{x}_{\mathrm{gfr}}=0.57$ gap units. Spline points: $(0,1),(0.75,1.01),(1.5,0.89)$.

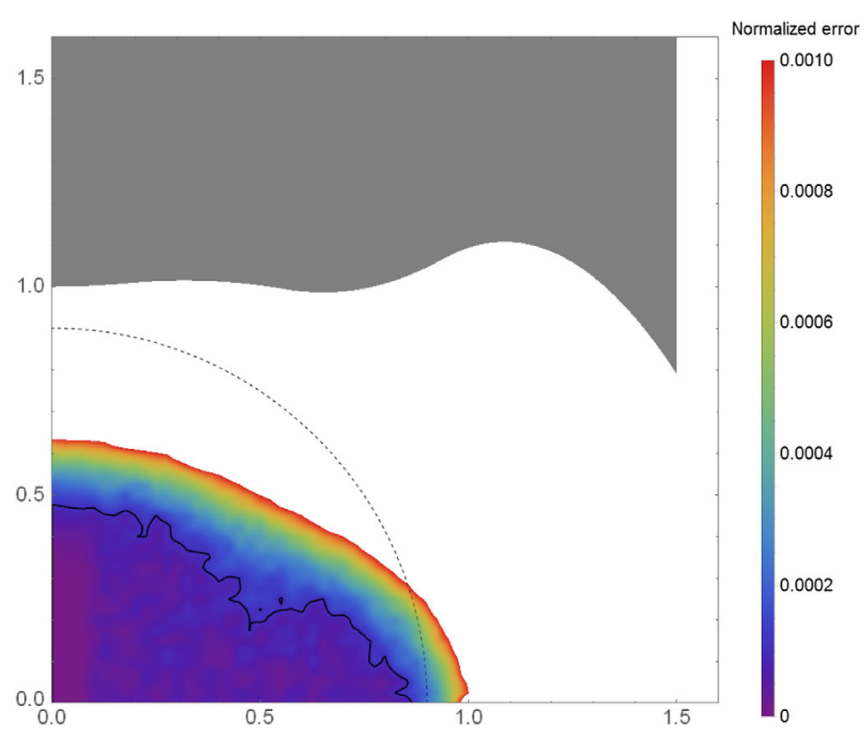

FIG. 56. 5 point spline. $\mathrm{x}_{\mathrm{gfr}}=0.85$ gap units. Spline points: $(0,1),(0.375,1.01),(0.75,1.00),(1.125,1.11),(1.5,0.79)$.

[4] M. Karppinen, N. Andreev, G. Apollinari, B. Auchmann, E. Barzi, R. Bossert, V. V. Kashikhin, A. Nobrega, I. Novitski, L. Rossi, D. Smekens, and A. V. Zlobin, Design of $11 \mathrm{~T}$ twin-aperture Nb3Sn dipole demonstrator magnet for LHC upgrades, IEEE Trans. Appl. Supercond. 22, 4901504 (2012).

[5] K. Halbach, Application of permanent magnets in accelerators and electron storage rings (invited), J. Appl. Phys. 57, 3605 (1985).

[6] A. C. Boxer, R. Bergmann, J. L. Ellsworth, D. T. Garnier, J. Kesner, M. E. Mauel, and P. Woskov, Turbulent inward pinch of plasma confined by a levitated dipole magnet, Nat. Phys. 6, 207 (2010). 
[7] E. Zavattini, G. Zavattini, G. Ruoso, E. Polacco, E. Milotti, M. Karuza, U. Gastaldi, G. Di Domenico, F. Della Valle, R. Cimino, S. Carusotto, G. Cantatore, and M. Bregant, Experimental Observation of Optical Rotation Generated in Vacuum by a Magnetic Field, Phys. Rev. Lett. 96, 110406 (2006).

[8] E. R. Andrew, Nuclear Magnetic Resonance (Cambridge University Press, Cambridge, England, 2009).

[9] G. Moresi and R. Magin, Miniature permanent magnet for table-top NMR, Concepts Magn. Reson., Part B 19B, 35 (2003).

[10] F. Cottone, H. Vocca, and L. Gammaitoni, Nonlinear Energy Harvesting, Phys. Rev. Lett. 102, 080601 (2009).

[11] A. Brahme, Design principles and clinical possibilities with a new generation of radiation therapy equipment: A review, Acta Oncologica 26, 403 (1987).

[12] D. Schardt, T. Elsässer, and D. Schulz-Ertner, Heavy-ion tumor therapy: Physical and radiobiological benefits, Rev. Mod. Phys. 82, 383 (2010).

[13] W. P. Leemans, P. Catravas, E. Esarey, C. G. R. Geddes, C. Toth, R. Trines, C. B. Schroeder, B. A. Shadwick, J. van Tilborg, and J. Faure, Electron-Yield Enhancement in a Laser-Wakefield Accelerator Driven by Asymmetric Laser Pulses, Phys. Rev. Lett. 89, 174802/1 (2002).

[14] W. P. Leemans, B Nagler, A. J. Gonsalves, Cs. Toth, K. Nakamura, C. G. R. Geddes, E. Esarey, C. B. Schroeder, and S M Hooker, GeV electron beams from a cm-scale accelerator, 2014, Nat. Phys. 2, 696 (2006).
[15] E. Esarey, C. B. Schroeder, and W. P. Leemans, Physics of laser-driven plasma-based electron accelerators, Rev. Mod. Phys. 81, 1229 (2009).

[16] J. H. Mathews and R. W. Howell, Complex Analysis: for Mathematics and Engineering, 6th ed. (Jones and Bartlett Pub. Inc., Sudbury, MA, 2012).

[17] E. T. Scharlemann, Wiggle plane focusing in linear wigglers, J. Appl. Phys. 58, 2154 (1985).

[18] J. S. Ryu, Y. Yao, C. S. Koh, and Y. J. Shin, 3-D optimal shape design of pole piece in permanent magnet MRI using parameterized nonlinear design sensitivity analysis, IEEE Trans. Magn. 42, 1351 (2006).

[19] A. Saleem, Design and optimization of a magnet pole for Magnetic Resonant Imaging, Ph.D. thesis, Aalto University, 2018.

[20] J. Kennedy and R. Eberhart, Particle swarm optimization, IEEE Int. Conf. Neural Networks 4, 1942 (1995).

[21] R. Holsinger and K. Halbach, Poisson Superfish, 2013, https://laacg.lanl.gov/laacg/services/download_sf .phtml.

[22] J. van Tilborg, S. K. Barber, F. Isono, C. B. Schroeder, E. Esarey, and W. P. Leemans, Free-electron lasers driven by laser plasma accelerators, AIP Conf. Proc. 1812, 020002 (2017).

[23] O. Chubar, P. Elleaume, and J. Chavanne, RADIA, 1997, http:/www.esrf.eu/Accelerators/Groups/InsertionDevices/ Software/Radia.

[24] R. Yamada and J. Moeller, Saturation effect and field correction using holes in yoke, Report No. TD-99-017, 1999. 